\title{
A SPITZER SURVEY OF PROTOPLANETARY DISK DUST IN THE YOUNG SERPENS CLOUD: HOW DO DUST CHARACTERISTICS EVOLVE WITH TIME?
}

\author{
Isa Oliveira ${ }^{1,2}$, Klaus M. Pontoppidan ${ }^{2}$, Bruno Merín ${ }^{3}$, Ewine F. van Dishoeck ${ }^{1,4}$, Fred Lahuis ${ }^{1,5}$, Vincent C. Geers ${ }^{6}$, \\ Jes K. Jørgensen ${ }^{7}$, Johan Olofsson ${ }^{8}$, Jean-Charles Augereau ${ }^{8}$, and Joanna M. Brown ${ }^{4}$ \\ ${ }^{1}$ Leiden Observatory, Leiden University, P.O. Box 9513, 2300 RA Leiden, The Netherlands; oliveira@ strw.leidenuniv.nl \\ ${ }^{2}$ California Institute of Technology, Division for Geological and Planetary Sciences, MS 150-21, Pasadena, CA 91125, USA \\ ${ }^{3}$ Herschel Science Center, European Space Agency (ESA), P.O. Box 78, 28691 Villanueva de la Cañada (Madrid), Spain \\ ${ }^{4}$ Max-Planck Institut für Extraterrestrische Physik, Giessenbachstrasse 1, 85748 Garching, Germany \\ ${ }^{5}$ SRON Netherlands Institute for Space Research, P.O. Box 800, 9700 AV Groningen, The Netherlands \\ ${ }^{6}$ University of Toronto, 50 St. George St., Toronto, ON M5R 2W9, Canada \\ ${ }^{7}$ Centre for Star and Planet Formation, Natural History Museum of Denmark, University of Copenhagen, Øster Voldgade 5-7, DK-1350 Copenhagen, Denmark \\ ${ }^{8}$ Laboratoire d'Astrophysique de Grenoble, Université Joseph Fourier, CNRS, UMR 5571, Grenoble, France \\ Received 2009 December 28; accepted 2010 March 16; published 2010 April 14
}

\begin{abstract}
We present Spitzer InfraRed Spectrograph (IRS) mid-infrared (5-35 $\mu \mathrm{m}$ ) spectra of a complete flux-limited sample $(\geqslant 3 \mathrm{mJy}$ at $8 \mu \mathrm{m}$ ) of young stellar object (YSO) candidates selected on the basis of their infrared colors in the Serpens Molecular Cloud. Spectra of 147 sources are presented and classified. Background stars (with slope consistent with a reddened stellar spectrum and silicate features in absorption), galaxies (with redshifted polycyclic aromatic hydrocarbon (PAH) features), and a planetary nebula (with high ionization lines) amount to $22 \%$ of contamination in this sample, leaving 115 true YSOs. Sources with rising spectra and ice absorption features, classified as embedded Stage I protostars, amount to $18 \%$ of the sample. The remaining $82 \%$ (94) of the disk sources are analyzed in terms of spectral energy distribution shapes, PAHs, and silicate features. The presence, strength, and shape of these silicate features are used to infer disk properties for these systems. About $8 \%$ of the disks have $30 / 13 \mu \mathrm{m}$ flux ratios consistent with cold disks with inner holes or gaps, and 3\% of the disks show PAH emission. Comparison with models indicates that dust grains in the surface of these disks have sizes of at least a few $\mu \mathrm{m}$. The $20 \mu \mathrm{m}$ silicate feature is sometimes seen in the absence of the $10 \mu \mathrm{m}$ feature, which may be indicative of very small holes in these disks. No significant difference is found in the distribution of silicate feature shapes and strengths between sources in clusters and in the field. Moreover, the results in Serpens are compared with other well-studied samples: the c2d IRS sample distributed over five clouds and a large sample of disks in the Taurus star-forming region. The remarkably similar distributions of silicate feature characteristics in samples with different environment and median ages_if significant-imply that the dust population in the disk surface results from an equilibrium between dust growth and destructive collision processes that are maintained over a few million years for any YSO population irrespective of environment.
\end{abstract}

Key words: circumstellar matter - infrared: stars - planetary systems - protoplanetary disks - stars: pre-main sequence

Online-only material: color figures

\section{INTRODUCTION}

Newly formed stars are observed to have infrared (IR) excess due to their circumstellar disk composed of dust and gas (Strom 1992; Hillenbrand 2008). Most older main-sequence (MS) stars, on the other hand, have photospheric emission with no excess in the IR. It is intuitive to conclude that the circumstellar disk evolves with time, gradually getting rid of the IR excess. One of the main questions in stellar astrophysics is how this happens.

Observational studies, as well as theoretical simulations, have demonstrated the interaction between star and disk. The stellar radiation facilitates disk evolution in terms of photoevaporation (e.g., Richling \& Yorke 2000; Alexander et al. 2006; Alexander 2008; Gorti \& Hollenbach 2009) or dust growth and settling (e.g., Weidenschilling 1980; Sterzik et al. 1995; Dominik \& Tielens 1997; Dullemond \& Dominik 2005; Brauer et al. 2007, 2008; Johansen et al. 2008). In the other direction, mass is accreted from the disk to the star following magnetic field lines (e.g., Muzerolle et al. 2003; White \& Basri 2003; Natta et al. 2004). A diversity of stellar temperatures, luminosities, and masses among young stars has been known and studied for decades. Facilitated by new IR and (sub-)millimeter observations, a great variety of disk shapes, structures, and masses is now being actively studied. The next step is to try to connect stellar and disk characteristics in order to understand the evolution of these systems.

The study of a single object, however, is unlikely to provide unambiguous information regarding the evolutionary stage of the associated disk. Most studies to date refer to samples of young stars scattered across the sky, or to sources distributed across large star-forming clouds like Taurus. In addition to evolutionary stage, the specific environment in which the stars are formed may influence the evolution of disks by dynamical and radiative interaction with other stars or through the initial conditions of the starting cloud, making it difficult to separate the evolutionary effects (e.g., Richling \& Yorke 1998, 2000). For this reason, clusters of stars are very often used as laboratories for calibrating the evolutionary sequence (e.g., Lada \& Lada 1995; Haisch et al. 2001). The power of this method, to gain statistical information on disk composition in coeval samples, was found to be very successful for loose associations of older, pre-MS stars such as the $8 \mathrm{Myr}$ old $\eta$ Cha (Bouwman et al. 
2006) and the 10 Myr old TW Hydrae association (Uchida et al. 2004). Identifying clusters of even younger disk populations is a natural step toward the completion of the empirical calibration of the evolution of disks surrounding young low-mass stars. This paper analyzes the inner disk properties of a flux-limited, complete unbiased sample of young stars with IR excess in the Serpens Molecular Cloud $(d=259 \pm 37 \mathrm{pc}$; Straizys et al. 1996) which has a mean age $\sim 5 \mathrm{Myr}$ (Oliveira et al. 2009) with a young stellar object (YSO) population in clusters and also in isolation. It has been recently argued (L. Loinard 2010, private communication) that the distance to Serpens could be considerably higher than previously calculated. This would imply a rather younger median age for this cloud.

The Spitzer Legacy Program "From Molecular Cores to Planet-Forming Disks" (c2d; Evans et al. 2003) has uncovered hundreds of objects with IR excess in five star-forming clouds (Cha II, Lupus, Ophiuchus, Perseus, and Serpens), and allowed statistical studies within a given cloud (Evans et al. 2009). The c2d study of Serpens with Infrared Array Camera (IRAC, 3.6, 4.5, 5.8 and $8.0 \mu \mathrm{m}$; Fazio et al. 2004) and MIPS (24 and $70 \mu \mathrm{m}$; Rieke et al. 2004) data has revealed a rich population of mostly previously unknown YSOs associated with IR excess, yielding a diversity of disk spectral energy distributions (SEDs; Harvey et al. 2006, 2007a, 2007b). Because of the compact area in Serpens mapped by Spitzer $\left(0.89 \mathrm{deg}^{2}\right)$, this impressive diversity of disks presents itself as an excellent laboratory for studies of early stellar evolution and planet formation. Indeed, the Serpens core (Cluster A, located in the northeastern part of the area studied by c2d) has been well studied in this sense (e.g., Zhang et al. 1988; Eiroa \& Casali 1992; Testi \& Sargent 1998; Kaas et al. 2004; Eiroa et al. 2005; Winston et al. 2007, 2009), whereas only some of the objects in Group C (formerly known as Cluster C) were studied with ISOCAM data (Djupvik et al. 2006).

Because of its wavelength coverage, sensitivity, and mapping capabilities, the Spitzer Space Telescope has offered an opportunity to study many of these systems (star+disk) in unprecedented detail. Spitzer's photometry in the mid-IR, where the radiation reprocessed by the dust is dominant, gives information on the shape of the disks and, indirectly, its evolutionary stage (assuming an evolution from flared to flat disks). Followup mid-IR spectroscopic observations with the InfraRed Spectrograph (IRS, 5-38 $\mu \mathrm{m}$; Houck et al. 2004) on board Spitzer probe the physical and chemical processes affecting the hot dust in the surface layers of the inner regions of the disk. The shapes and strengths of the silicate features provide information on dust grain size distribution and structure (e.g., van Boekel et al. 2003; Przygodda et al. 2003; Bouwman et al. 2008; KesslerSilacci et al. 2006, 2007; Geers et al. 2006; Watson et al. 2009; Olofsson et al. 2009). These, in turn, reflect dynamical processes such as radial and vertical mixing, and physical processes such as annealing. A smooth strong $\mathrm{Si}-\mathrm{O}$ stretching mode feature centered at $9.8 \mu \mathrm{m}$ is indicative of small amorphous silicates (like those found in the interstellar medium (ISM)) while a structured weaker and broader feature reveals bigger grains or the presence of crystalline silicates. Polycyclic aromatic hydrocarbon (PAH) features are a probe of the UV radiation incident on the disk, whereas their abundance plays a crucial role in models of disk heating and chemistry (e.g., Geers et al. 2006; Dullemond et al. 2007; Visser et al. 2007). The shape and slope of the mid-infrared excess provides information on the flaring geometry of the disks (Dullemond \& Dominik 2004), while ice bands may form for highly inclined sources (edge-on) where the light from the central object passes through the dusty material in the outer parts of the disk (Pontoppidan et al. 2005). Thus, the wavelength range probed by the IRS spectra enables analysis of the geometry of individual disks. It also probes the temperature and dust size distributions as well as crystallinity of dust in the disk surface at radii of 0.1-few AU. Statistical results from a number of sources help the understanding of the progression of disk clearing and possibly planet formation.

Our group has been conducting multi-wavelength observing campaigns of Serpens. Optical and near-IR wavelength data, where the stellar radiation dominates, are being used to characterize the central sources of these systems (Oliveira et al. 2009). Effective temperatures, luminosities, extinctions, mass accretion rates, as well as relative ages and masses are being determined. In this paper, we present a complete flux-limited set of Spitzer IRS spectra for this previously unexplored young stellar population in Serpens. We analyze these spectra in terms of common and individual characteristics and compare the results to those of Taurus, one of the best studied molecular clouds to date and dominated by isolated star formation. A subsequent paper will deal with the full SED fitting for the disk sources in Serpens and their detailed analyses. Our ultimate goal is to statistically trace the evolution of young low-mass stars by means of the spectroscopic signatures of disk evolution, discussed above.

Section 2 describes our Spitzer IRS data. In Section 3, we divide our sample into categories based on their IRS spectra: the background contaminants are presented in Section 3.1 separated according to the nature of the objects; embedded sources are presented in Section 3.2, and disk sources in Section 3.3 (with emphasis on PAH and silicate emission). In Section 4, we discuss disk properties in relation to environment and to another cloud, Taurus, for comparison. In Section 5, we present our conclusions.

\section{SPITZER IRS DATA}

\subsection{Sample Selection}

Harvey et al. (2007b) describe the selection criteria used by the c2d team to identify YSO candidates based on the Spitzer IRAC and MIPS data, band-merged with the Two Micron All Sky Survey (2MASS) catalog. They used a number of color-color and color-magnitude diagrams to separate YSOs (both embedded YSOs and young stars with disks) from other types of sources, such as background galaxies and stars. In this manner, a set of 235 YSOs was identified in Serpens, in both clusters and in isolation, as described in Section 4.1. These criteria, however, are not fail-proof. Harvey et al. (2007b) treated this problem very carefully in a statistical sense, but the locus region for YSOs and, for instance, background galaxies overlap somewhat in color-color diagrams with Spitzer photometry. Therefore, some contamination may well still be present in the sample which was impossible to disentangle from photometry alone.

An additional criterion was applied to this sample in order to guarantee IRS spectra with sufficient quality to allow comparative studies of solid-state features, namely signal-to-noise ratio $(\mathrm{S} / \mathrm{N}) \geqslant 30$ on the continuum. A lower limit flux cutoff of $3 \mathrm{mJy}$ at $8 \mu \mathrm{m}$ was imposed. This flux limit ensures coverage down to the brown dwarf limit $\left(L \sim 0.01 L_{\odot}\right)$ and leads to a final sample of 147 objects, the same as in Oliveira et al. (2009). This is a complete flux-limited sample of IR excess sources in the c2d mapped area, except in the Serpens core. It is also important 


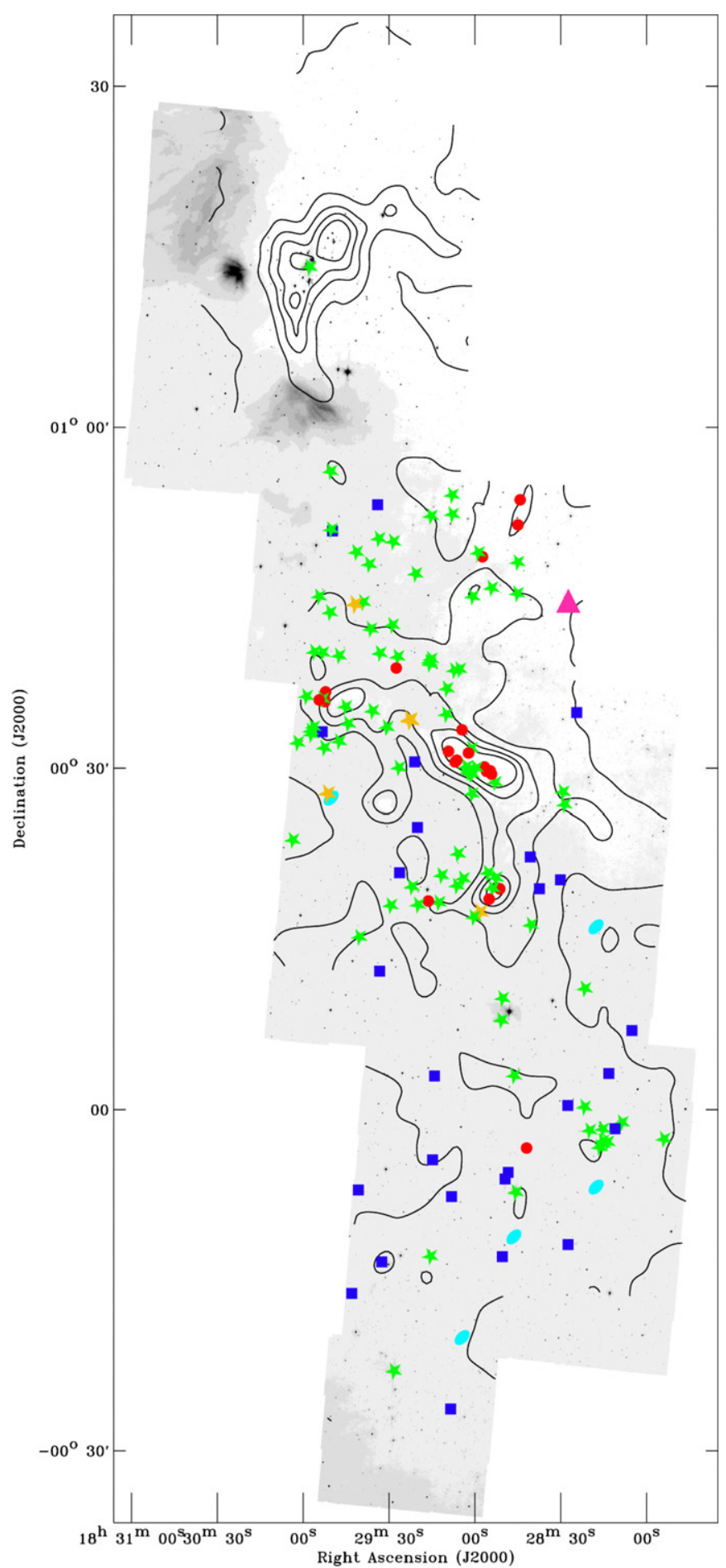

Figure 1. Observed objects overplotted on the c2d IRAC4 $(8.0 \mu \mathrm{m})$ map of Serpens. The contours $(5,10,15,20$, and $25 \mathrm{mag})$ of visual extinction are derived from the c2d extinction maps (Evans et al. 2007). Blue squares are background stars, cyan ellipses are redshifted galaxies, red circles are embedded sources, and stars represent disks (yellow disks with PAH emission and green other disk sources). The pink triangle is the PN candidate (see Section A.3 for details).

(A color version of this figure is available in the online journal.)

to note that this sample, by definition, does not include young stars without IR excess (Class III). Figure 1 shows the distribution of our observed objects in the Serpens Molecular Cloud. Table 1 lists the sample together with their $2-24 \mu \mathrm{m}$ spectral slope.

\subsection{Observations and Data Reduction}

Out of the 147 objects in this sample, 22 were observed between 2004 September and 2006 June, as part of the c2d IRS 2nd Look campaign. The reduced high resolution spectra $(R=$ $\lambda / \Delta \lambda=600$; Short High (SH), 9.9-19.6 $\mu \mathrm{m}$ and Long High (LH), 18.7-37.2 $\mu \mathrm{m}$ ), complemented with Short Low spectra (SL, $R=50-100 ; 5.2-14.5 \mu \mathrm{m}$ ) are public to the community through the $\mathrm{c} 2 \mathrm{~d}$ delivery web Site (Evans et al. 2007). The other 125 targets were observed as part of Spitzer Space Telescope's cycle 3 program (GO3 30223, PI: Pontoppidan) in the low resolution module (SL and Long Low (LL), 14.0-38.0 $\mu \mathrm{m}$ ) in 35 acousto-optical spectrometers (AORs), between 2006 October and 2007 April.

All objects were observed in IRS staring mode and extracted from the SSC pipeline (version S12.0.2 for the c2d IRS 2nd look program, and version $\mathrm{S} 15.3 .0$ for the GO3 program) basic calibration data (BCD) using the $\mathrm{c} 2 \mathrm{~d}$ reduction pipeline (Lahuis et al. 2006). This process includes bad-pixel correction, optimal point-spread function (PSF) aperture extraction, defringing, and order matching. Technical information about each object can be found in Table 1.

\section{RESULTS}

The large number of objects studied here allows statistical studies of each stage of disk evolution in a single star-forming region. By simply looking at the spectra, a diversity of spectral shapes can be noted immediately. This diversity of objects can be divided into several categories, each of which is thought to be related to a different evolutionary stage of the star+disk system, described below.

\subsection{Background Sources}

Due to the low galactic latitude of Serpens $(l=030.4733, b=$ +05.1018 ) the contamination by galactic background sources is expected to be higher for this region than for other studied starforming regions (e.g., Taurus, Lupus, Chamaeleon). With the help of the IRS spectra, background contaminants can readily be identified, even when seen through a large column density of molecular cloud material. These are presented and discussed in the Appendix.

To further study the YSOs, the 32 contaminants are removed from the sample, which then consists of 115 objects for further analysis.

\subsection{Embedded Sources}

A newly formed protostar, still embedded in an envelope of gas and dust, will show an excess in the mid-IR that rises with wavelength. In this cold environment, a significant fraction of the molecules are found in ices. Many of these ices are observed as absorption features in the wavelength range of the IRS instrument (e.g., Boogert et al. 2004).

Of our sample, 21 sources have spectra consistent with embedded sources. These spectra are shown in Figure 2. The most prominent ice feature, $\mathrm{CO}_{2}$ at $15.2 \mu \mathrm{m}$, can readily be seen in all spectra. Weaker features, such as $\mathrm{H}_{2} \mathrm{O}$ at 6.0 and $6.85 \mu \mathrm{m}$ and $\mathrm{CH}_{3} \mathrm{OH}$ at $9.7 \mu \mathrm{m}$, need high $\mathrm{S} / \mathrm{N}$. Those features are detected in objects $26,27,28,44,45,47,49,67,72,73,135$, and 140. As can be seen in Figure 1, the embedded objects seem to be concentrated in high extinction regions $\left(A_{V} \sim 10-15 \mathrm{mag}\right)$.

The embedded objects observed as part of the c2d IRS 2nd Look Program have been studied in detail in a series of papers 
Table 1

Observed Objects in Serpens

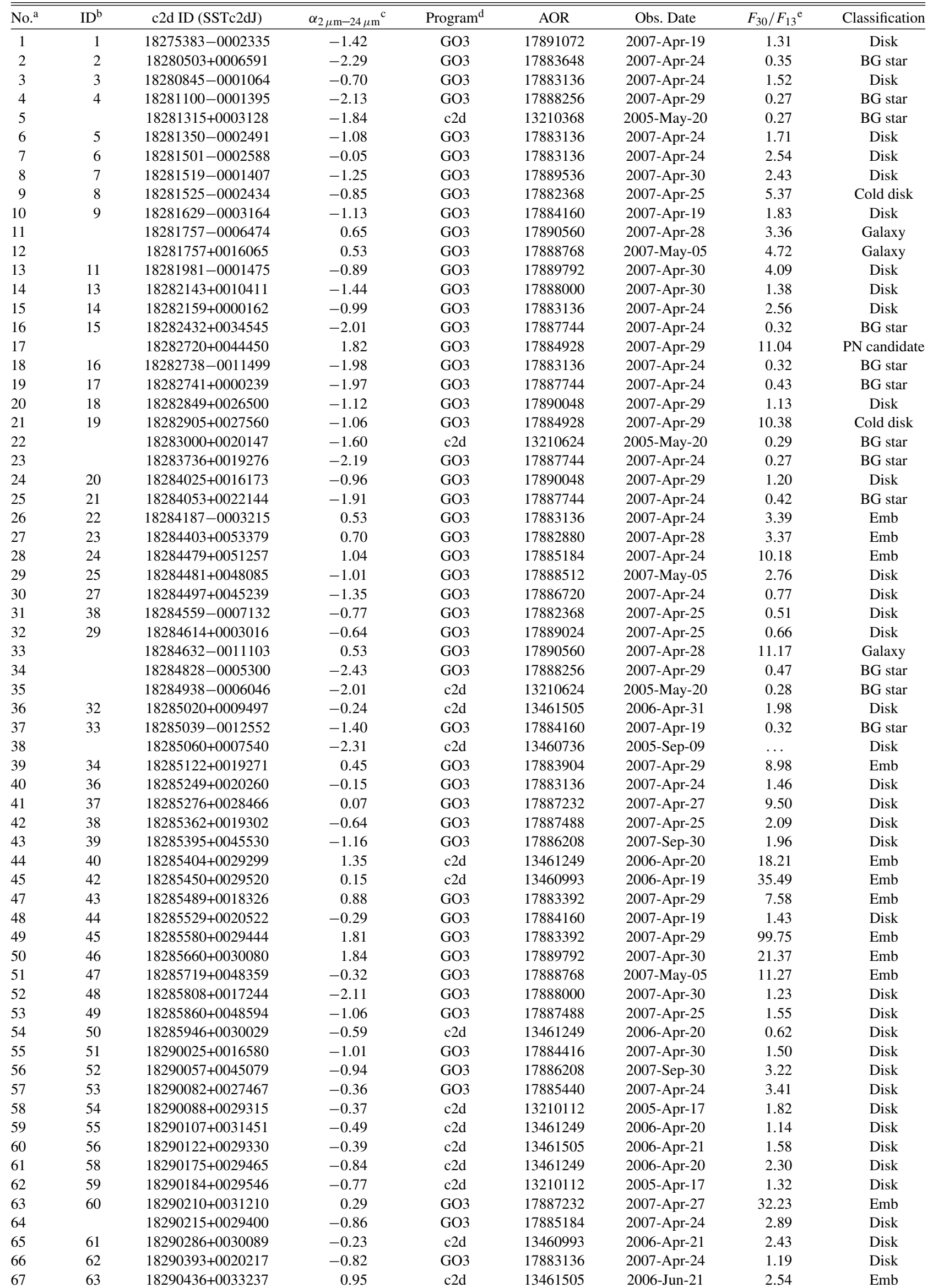


Table 1

(Continued)

\begin{tabular}{|c|c|c|c|c|c|c|c|c|}
\hline No. ${ }^{a}$ & $\mathrm{ID}^{\mathrm{b}}$ & c2d ID (SSTc2dJ) & $\alpha_{2 \mu \mathrm{m}-24 \mu \mathrm{m}^{\mathrm{c}}}$ & Program $^{\mathrm{d}}$ & AOR & Obs. Date & $F_{30} / F_{13}{ }^{\mathrm{e}}$ & Classification \\
\hline 68 & & $18290442-0020018$ & 0.52 & GO3 & 17890560 & 2007-Apr-28 & 5.61 & Galaxy \\
\hline 69 & 64 & $18290518+0038438$ & -1.24 & GO3 & 17889792 & 2007-Apr-30 & 5.64 & Cold disk \\
\hline 70 & 65 & $18290575+0022325$ & -2.12 & GO3 & 17886976 & 2007-Apr-29 & 1.76 & Disk \\
\hline 71 & 66 & $18290615+0019444$ & -1.52 & GO3 & 17888512 & 2007-May-05 & 2.49 & Disk \\
\hline 72 & 67 & $18290620+0030430$ & 1.69 & $\mathrm{c} 2 \mathrm{~d}$ & 13461249 & 2006-Apr-21 & 17.07 & Emb \\
\hline 73 & 68 & $18290680+0030340$ & 1.67 & $\mathrm{c} 2 \mathrm{~d}$ & 13460993 & 2006-Apr-21 & 75.97 & Emb \\
\hline 74 & 69 & $18290699+0038377$ & -0.63 & GO3 & 17884416 & 2007-Apr-30 & 2.90 & Disk \\
\hline 75 & 71 & $18290765+0052223$ & -1.01 & GO3 & 17888512 & 2007-May-05 & 2.01 & Disk \\
\hline 76 & 72 & $18290775+0054037$ & -1.06 & GO3 & 17888000 & 2007-Apr-30 & 1.34 & Disk \\
\hline 77 & 73 & $18290808-0007371$ & -2.22 & GO3 & 17888256 & 2007-Apr-29 & 0.27 & BG star \\
\hline 78 & & $18290843-0026187$ & -2.28 & GO3 & 17885696 & 2007-Apr-28 & 0.31 & BG star \\
\hline 79 & 75 & $18290910+0031300$ & 0.24 & GO3 & 17884928 & 2007-Apr-29 & 51.02 & Emb \\
\hline 80 & 76 & $18290956+0037016$ & -1.13 & GO3 & 17882368 & 2007-Apr-25 & 0.51 & Disk \\
\hline 81 & 77 & $18290980+0034459$ & -0.97 & $\mathrm{c} 2 \mathrm{~d}$ & 13210624 & 2005-May-20 & 0.85 & Disk \\
\hline 82 & 78 & $18291148+0020387$ & -1.34 & GO3 & 17887232 & 2007-Apr-27 & 11.90 & Cold disk \\
\hline 83 & 79 & $18291249+0018152$ & -1.06 & GO3 & 17889536 & 2007-Apr-30 & 2.06 & Disk \\
\hline 84 & 81 & $18291407+0002589$ & -2.19 & GO3 & 17885696 & 2007-Apr-28 & 0.30 & BG star \\
\hline 85 & & $18291477-0004237$ & -2.29 & $\mathrm{c} 2 \mathrm{~d}$ & 13210112 & 2005-Apr-17 & 0.24 & BG star \\
\hline 86 & 83 & $18291508+0052124$ & -1.89 & GO3 & 17882880 & 2007-Apr-28 & 0.45 & Disk \\
\hline 87 & 84 & $18291513+0039378$ & -1.50 & GO3 & 17889024 & 2007-Apr-25 & 1.01 & Disk \\
\hline 88 & 85 & $18291539-0012519$ & -1.72 & GO3 & 17885952 & 2006-Oct-23 & 0.50 & Disk \\
\hline 89 & 86 & $18291557+0039119$ & -0.56 & GO3 & 17884416 & 2007-Apr-30 & 2.51 & Disk \\
\hline 90 & 87 & $18291563+0039228$ & -0.89 & GO3 & 17885184 & 2007-Apr-24 & 6.52 & Cold disk \\
\hline 91 & 88 & $18291617+0018227$ & 0.45 & $\mathrm{c} 2 \mathrm{~d}$ & 13210112 & 2005-Apr-17 & 2.11 & $\mathrm{Emb}$ \\
\hline 92 & 89 & $18291969+0018031$ & -1.23 & GO3 & 17885440 & 2007-Apr-24 & 2.10 & Disk \\
\hline 93 & 90 & $18292001+0024497$ & -2.04 & GO3 & 17886720 & 2007-Apr-24 & 0.29 & BG star \\
\hline 94 & & $18292050+0047080$ & 1.29 & GO3 & 17888768 & 2007-May-05 & 25.76 & Disk \\
\hline 95 & 93 & $18292094+0030345$ & -1.73 & GO3 & 17882880 & 2007-Apr-28 & 0.40 & BG star \\
\hline 96 & 94 & $18292184+0019386$ & -0.99 & GO3 & 17886976 & 2007-Apr-29 & 1.54 & Disk \\
\hline 97 & & $18292250+0034118$ & -1.65 & GO3 & 17889280 & 2007-Apr-24 & 0.29 & Disk \\
\hline 98 & & $18292253+0034176$ & -2.48 & GO3 & 17889024 & 2007 Apr 25 & 1.34 & Disk \\
\hline 99 & 95 & $18292616+0020518$ & -2.26 & GO3 & 17883648 & 2007-Apr-24 & 0.37 & BG star \\
\hline 100 & 96 & $18292640+0030042$ & -0.98 & GO3 & 17888768 & 2007-May-5 & 1.89 & Disk \\
\hline 101 & 97 & $18292679+0039497$ & -0.60 & GO3 & 17885440 & 2007-Apr-24 & 4.13 & Disk \\
\hline 102 & 99 & $18292736+0038496$ & 0.29 & GO3 & 17886464 & 2007-Apr-29 & 3.95 & Emb \\
\hline 103 & 100 & $18292824-0022574$ & -0.41 & $\mathrm{c} 2 \mathrm{~d}$ & 13210368 & 2005-May-20 & 1.17 & Disk \\
\hline 104 & & $18292833+0049569$ & -0.48 & GO3 & 17887488 & 2007-Apr-25 & 1.85 & Disk \\
\hline 105 & & $18292864+0042369$ & -0.97 & GO3 & 17890048 & 2007-Apr-29 & 1.94 & Disk \\
\hline 106 & 102 & $18292927+0018000$ & -1.24 & GO3 & 17889792 & 2007-Apr-30 & 1.15 & Disk \\
\hline 107 & & $18293056+0033377$ & -0.60 & GO3 & 17889536 & 2007-Apr-30 & 1.46 & Disk \\
\hline 108 & 105 & $18293254-0013233$ & -2.58 & GO3 & 17884672 & 2007-Apr-28 & 1.36 & BG star \\
\hline 109 & 106 & $18293300+0040087$ & -1.35 & GO3 & 17888512 & 2007-May-5 & 2.16 & Disk \\
\hline 110 & 107 & $18293319+0012122$ & -1.81 & GO3 & 17883648 & 2007-Apr-24 & 0.34 & BG star \\
\hline 111 & & $18293337+0050136$ & -0.26 & GO3 & 17888768 & 2007-May-5 & 3.80 & Disk \\
\hline 112 & 109 & $18293381+0053118$ & -2.06 & GO3 & 17886720 & 2007-Apr-24 & 0.29 & BG star \\
\hline 113 & 110 & $18293561+0035038$ & -1.53 & GO3 & 17883904 & 2007-Apr-29 & 5.76 & Cold disk \\
\hline 114 & 111 & $18293619+0042167$ & -0.92 & GO3 & 17883904 & 2007-Apr-29 & 7.52 & Cold disk \\
\hline 115 & 114 & $18293672+0047579$ & -1.00 & GO3 & 17886976 & 2007-Apr-29 & 3.19 & Disk \\
\hline 116 & 116 & $18293882+0044380$ & -1.23 & GO3 & 17890048 & 2007-Apr-29 & 1.55 & Disk \\
\hline 117 & 118 & $18294020+0015131$ & 0.87 & GO3 & 17883392 & 2007-Apr-29 & 2.72 & Disk \\
\hline 118 & & $18294067-0007033$ & -2.53 & GO3 & 17884672 & 2007-Apr-28 & 0.38 & BG star \\
\hline 119 & 119 & $18294121+0049020$ & -1.39 & GO3 & 17889280 & 2007-Apr-24 & 1.55 & Disk \\
\hline 120 & 123 & $18294168+0044270$ & -1.42 & GO3 & 17884416 & 2007-Apr-30 & 3.13 & Disk \\
\hline 121 & & $18294301-0016083$ & -2.18 & GO3 & 17890304 & 2006-Oct-25 & 0.33 & BG star \\
\hline 122 & 126 & $18294410+0033561$ & -1.68 & GO3 & 17886464 & 2007-Apr-29 & 10.92 & Cold disk \\
\hline 123 & 129 & $18294503+0035266$ & -1.26 & GO3 & 17886976 & 2007-Apr-29 & 3.71 & Disk \\
\hline 124 & 132 & $18294725+0039556$ & -1.32 & GO3 & 17887232 & 2007-Apr-27 & 2.22 & Disk \\
\hline 125 & 133 & $18294726+0032230$ & -1.31 & GO3 & 17886976 & 2007-Apr-29 & 1.50 & Disk \\
\hline 126 & 140 & $18294962+0050528$ & -2.00 & GO3 & 17890816 & 2007-Apr-24 & 0.35 & BG star \\
\hline 127 & 143 & $18295001+0051015$ & -1.68 & GO3 & 17889280 & 2007-Apr-24 & 1.23 & Disk \\
\hline 128 & & $18295012+0027229$ & 0.14 & GO3 & 17888768 & 2007-May-5 & 2.60 & Galaxy \\
\hline 129 & 144 & $18295016+0056081$ & -0.76 & GO3 & 17886208 & 2007-Sep-30 & 1.86 & Disk \\
\hline 130 & 145 & $18295041+0043437$ & -1.32 & GO3 & 17886720 & 2007-Apr-24 & 2.14 & Disk \\
\hline 131 & 148 & $18295130+0027479$ & -2.42 & GO3 & 17889024 & 2007-Apr-25 & 2.09 & Disk \\
\hline 132 & 149 & $18295206+0036436$ & -0.06 & GO3 & 17886464 & 2007-Apr-29 & 4.04 & Emb \\
\hline 133 & & $18295240+0035527$ & -0.33 & GO3 & 17884416 & 2007-Apr-30 & 3.07 & Emb \\
\hline
\end{tabular}


Table 1

(Continued)

\begin{tabular}{|c|c|c|c|c|c|c|c|c|}
\hline No. ${ }^{a}$ & $\mathrm{ID}^{\mathrm{b}}$ & c2d ID (SSTc2dJ) & $\alpha_{2 \mu \mathrm{m}-24 \mu \mathrm{m}^{\mathrm{c}}}$ & Program $^{\mathrm{d}}$ & AOR & Obs. Date & $F_{30} / F_{13} \mathrm{e}^{\mathrm{e}}$ & Classification \\
\hline 134 & 153 & $18295244+0031496$ & -0.59 & GO3 & 17889792 & 2007-Apr-30 & 1.48 & Disk \\
\hline 135 & 154 & $18295252+0036116$ & 0.78 & GO3 & 17883392 & 2007-Apr-29 & 24.39 & Emb \\
\hline 136 & 156 & $18295304+0040105$ & -1.16 & GO3 & 17889536 & 2007-Apr-30 & 1.50 & Disk \\
\hline 137 & 157 & $18295305+0036065$ & 0.07 & GO3 & 17882624 & 2006-Oct-23 & 10.57 & Disk \\
\hline 138 & 161 & $18295322+0033129$ & -1.99 & GO3 & 17886720 & 2007-Apr-24 & 2.35 & BG star \\
\hline 139 & 165 & $18295422+0045076$ & -2.35 & GO3 & 17889024 & 2007-Apr-25 & 1.03 & Disk \\
\hline 140 & 166 & $18295435+0036015$ & -0.19 & GO3 & 17887232 & 2007-Apr-27 & 9.65 & Emb \\
\hline 142 & 172 & $18295592+0040150$ & -0.85 & GO3 & 17888000 & 2007-Apr-30 & 1.21 & Disk \\
\hline 143 & 173 & $18295620+0033391$ & -0.54 & GO3 & 17889024 & 2007-Apr-25 & 1.21 & Disk \\
\hline 144 & 177 & $18295701+0033001$ & -1.15 & GO3 & 17882880 & 2007-Apr-28 & 0.96 & Disk \\
\hline 145 & 178 & $18295714+0033185$ & -0.82 & GO3 & 17883648 & 2007-Apr-24 & 1.26 & Disk \\
\hline 146 & 182 & $18295772+0114057$ & -0.44 & $\mathrm{c} 2 \mathrm{~d}$ & 9828352 & 2004-Sep-02 & 1.41 & Disk \\
\hline 147 & 189 & $18295872+0036205$ & -1.11 & $\mathrm{GO} 3$ & 17888000 & 2007-Apr-30 & 0.72 & Disk \\
\hline 148 & 206 & $18300178+0032162$ & -1.25 & GO3 & 17886976 & 2007-Apr-29 & 1.90 & Disk \\
\hline 149 & 210 & $18300350+0023450$ & -1.22 & GO3 & 17888000 & 2007-Apr-30 & 1.81 & Disk \\
\hline
\end{tabular}

Notes.

a As in Oliveira et al. (2009).

${ }^{\mathrm{b}}$ From Harvey et al. (2007b).

c $\frac{d \log \left(\lambda F_{\lambda}\right)}{d \log (\lambda)}$ between the 2MASS $K(2 \mu \mathrm{m})$ and the MIPS1 $(24 \mu \mathrm{m})$ bands (Harvey et al. 2007b).

d "GO3" means the object was observed as part of the Spitzer Space Telescope's cycle 3 program 30223, PI: K. Pontoppidan; "c2d" means the object was observed as part of the $\mathrm{c} 2 \mathrm{~d} 2$ nd Look program, PI: N. Evans.

${ }^{\mathrm{e}}$ Flux ratio between 30 and $13 \mu \mathrm{m}$.
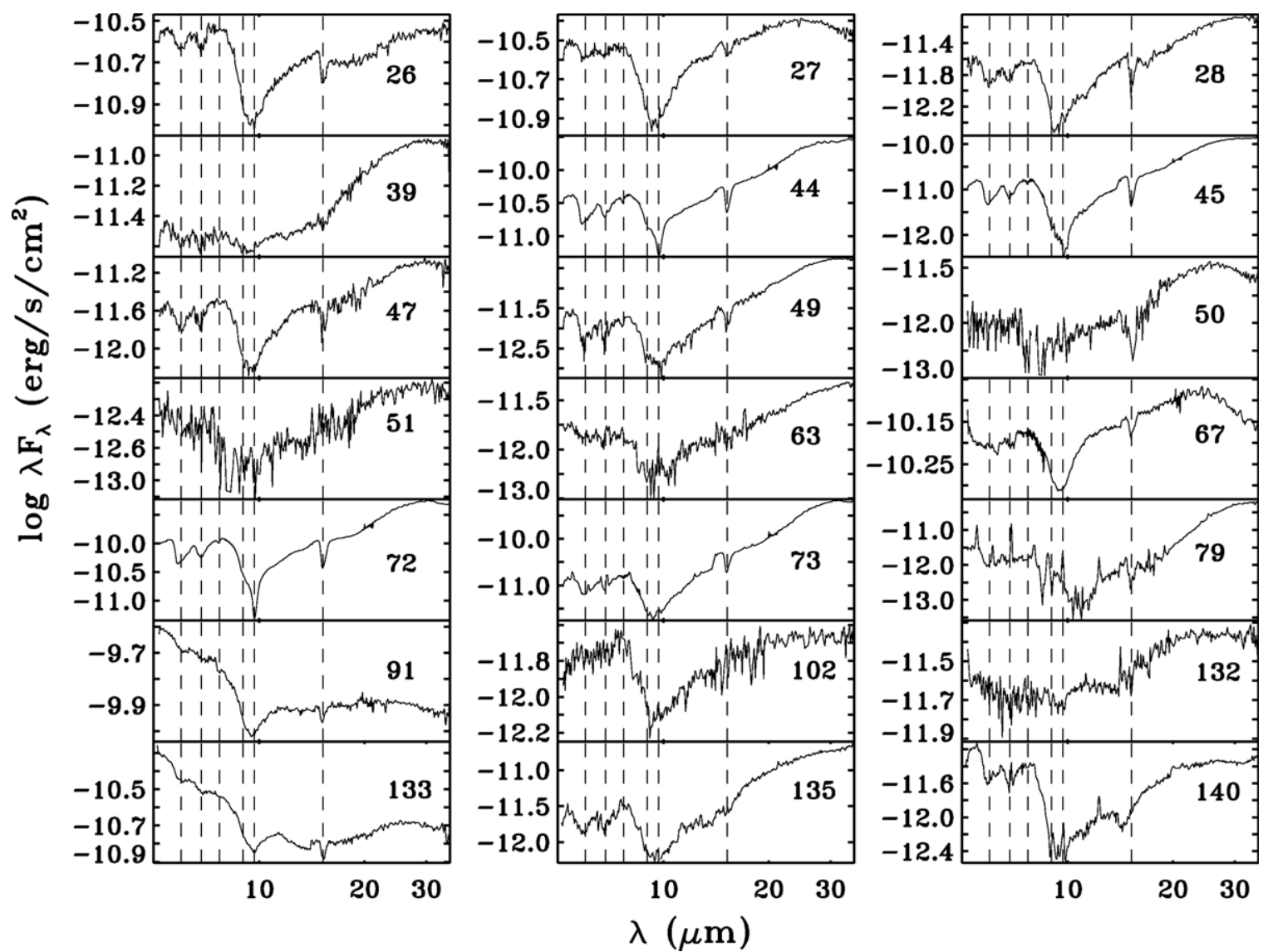

Figure 2. IRS spectra of the embedded sources in Serpens with rising mid-IR spectra. The prominent ice absorption features (at 6.0, 6.85, 7.7, 9.0, and 15.2 $\mu \mathrm{m}$ ) are marked, together with the silicate absorption feature at $9.7 \mu \mathrm{m}$. The object number is indicated at the top right of each panel.

on ices around YSOs $\left(\mathrm{H}_{2} \mathrm{O}\right.$ in Boogert et al. 2008; $\mathrm{CO}_{2}$ in Pontoppidan et al. 2008; $\mathrm{CH}_{4}$ at $7.7 \mu \mathrm{m}$ in Öberg et al. 2008; and $\mathrm{NH}_{3}$ at $9.0 \mu \mathrm{m}$ and $\mathrm{CH}_{3} \mathrm{OH}$ in Bottinelli et al. 2010) and are not discussed further in this paper.

\subsection{Disk Sources}

YSOs with IR excess that have a flat or negative slope in the near- to mid-IR are identified as disk sources. Following Greene 


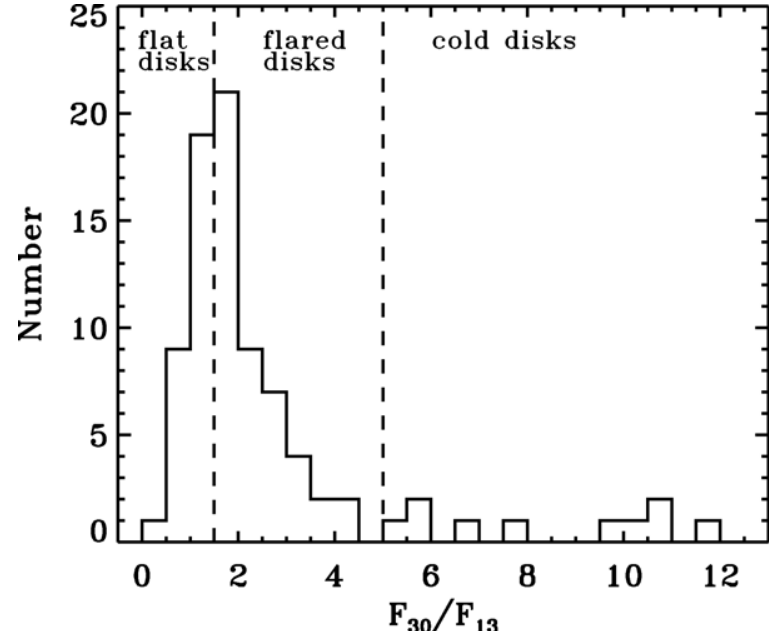

Figure 3. Distribution of the flux ratio between 30 and $13 \mu \mathrm{m}$, used as a proxy for disk geometry.

et al. (1994) and Evans et al. (2009), flat sources have $-0.3 \leqslant$ $\alpha_{2 \mu \mathrm{m}-24 \mu \mathrm{m}} \leqslant 0.3$, Class II sources have $-1.6 \leqslant \alpha_{2 \mu \mathrm{m}-24 \mu \mathrm{m}} \leqslant$ -0.3 , and Class III sources have $\alpha_{2 \mu \mathrm{m}-24 \mu \mathrm{m}} \leqslant-1.6$. Many of these sources also have silicate emission bands and a few show PAH features. In the following, these different features are analyzed and discussed in detail for the total of 94 disk sources.

\subsubsection{Disk Geometry: $F_{30} / F_{13}$ and Cold Disks}

Disk geometry is inferred from the flux ratio between 30 and $13 \mu \mathrm{m}\left(F_{30} / F_{13}\right)$. High ratios $\left(F_{30} / F_{13} \gtrsim 15\right)$ yield edge-on disks, intermediate values $\left(5 \lesssim F_{30} / F_{13} \lesssim 15\right.$ and $\left.1.5 \lesssim F_{30} / F_{13} \lesssim 5\right)$ identify cold disks and flared disks with considerable excess in the IR, respectively, and low ratios $\left(F_{30} / F_{13} \lesssim 1.5\right)$ select flat, settled disks with little IR excess (Brown et al. 2007; Merín et al. 2010). Figure 3 shows the distribution of $F_{30} / F_{13}$ for the disk population in Serpens.

Cold disks, sometimes referred to as transitional disks, as studied in Brown et al. (2007) and Merín et al. (2010), present a peculiar SED, with inner gaps or holes producing a region with no IR excess in the near- to mid-IR but a substantial excess at mid- to far-IR wavelengths. Binaries (Ireland \& Kraus 2008), planet formation (Quillen et al. 2004; Varnière et al. 2006) and photoevaporation (Clarke et al. 2001; Alexander et al. 2006) are some of the suggested mechanisms for this removal of warm dust.
Following Brown et al. (2007), $F_{30} / F_{13}$ is used to differentiate cold disks from the general disk population. Those wavelengths were chosen to avoid the silicate features and to probe the spectra steeply rising at mid- to far-IR following a deficit at shorter wavelengths, characteristic of cold disks. Eight objects $(9,21$, $69,82,90,113,114$, and 122) in this sample are classified as cold disks based on $5 \lesssim F_{30} / F_{13} \lesssim 15$, corresponding to $8.5 \%$ of the disk population. These spectra are shown in Figure 4. For clarity, the original spectra are shown in gray, while in black a binned version is overplotted. The binning was done using a Savitzky-Golay (Savitzky \& Golay 1964) filter of order 0 and degree 3 , for illustrative purposes only. This is the most unbiased survey of cold disks to date, based entirely on spectroscopy in the critical mid-IR range, and should thus give a representative fraction of such disks. These objects are also part of a larger cold disk sample, studied by Merín et al. (submitted), where the authors have selected cold disks from photometric criteria in all five $\mathrm{c} 2 \mathrm{~d}$ clouds.

\subsubsection{PAH Emission}

PAHs are large molecules whose emission is excited by UV radiation out to large distances from the star. Thus, these features are good diagnostics of the amount of stellar UV intercepted by the disk surface.

Almost half of the sample of Herbig Ae/Be stars (young stars of intermediate masses) studied by Meeus et al. (2001) and Acke \& van den Ancker (2004) show PAH emission. Geers et al. (2006), on the other hand, show that PAH emission is not as common among low-mass YSOs as it is for more massive stars. The authors argue that the lack of features is not only due to their weaker UV radiation fields, but also to a lower PAH abundance compared with the general ISM.

In the current sample, five sources have clear PAH emission, presented in Figure 5. Although a great variation in shapes and strengths is seen, all sources show the features at 6.2, 7.7, 8.6, 11.2, and $12.8 \mu \mathrm{m}$. Objects 52, 97, and 131 also show a feature that could be the PAH band at $16.4 \mu \mathrm{m}$, although the feature does not match perfectly in position.

The central stars in these five sources were studied with optical spectroscopy in Oliveira et al. (2009). Spectral types and luminosities were derived, yielding ages and masses for each object. Objects 52, 98, 120, and 131 have masses between 1.9 and $2.7 M_{\odot}$ (spectral type G3 to A2), while object 97 has lower mass, of around $0.45 M_{\odot}$ (M2). Objects 97 and 98 are spatially very close to each other and have very similar PAH features. IRAC Band $4(8 \mu \mathrm{m})$ images show that there is
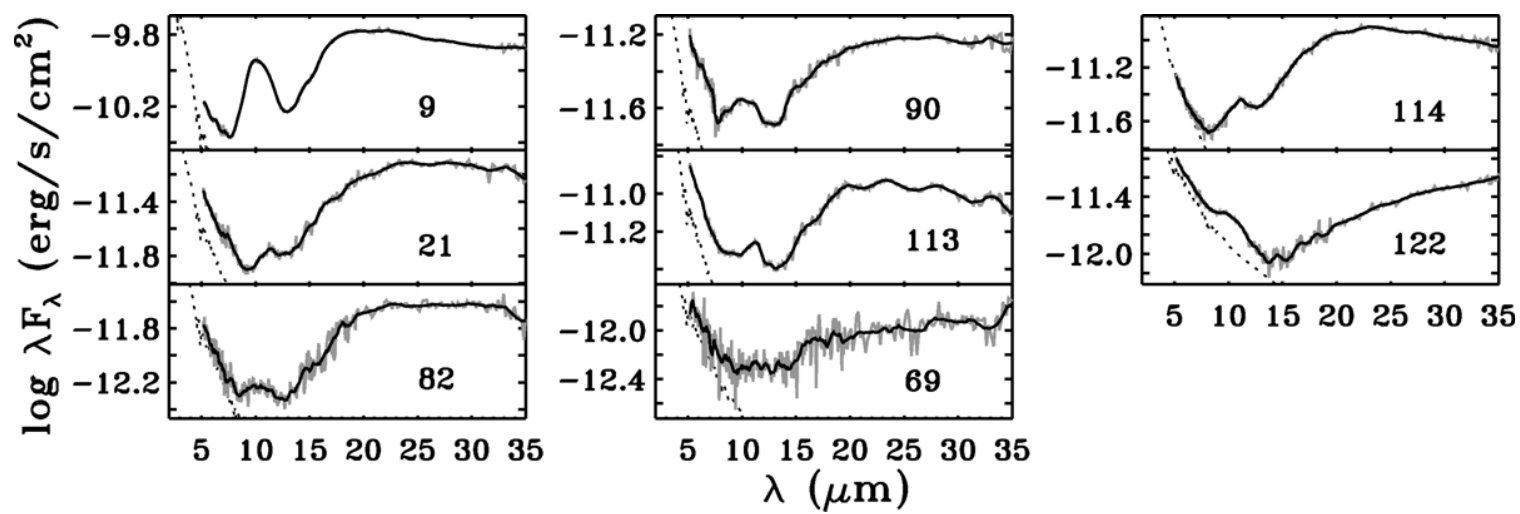

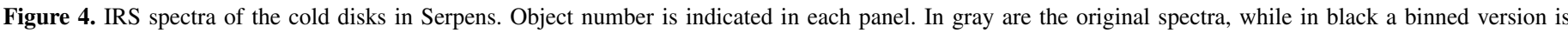
overplotted. The dotted line represents the stellar photosphere, for comparison. 


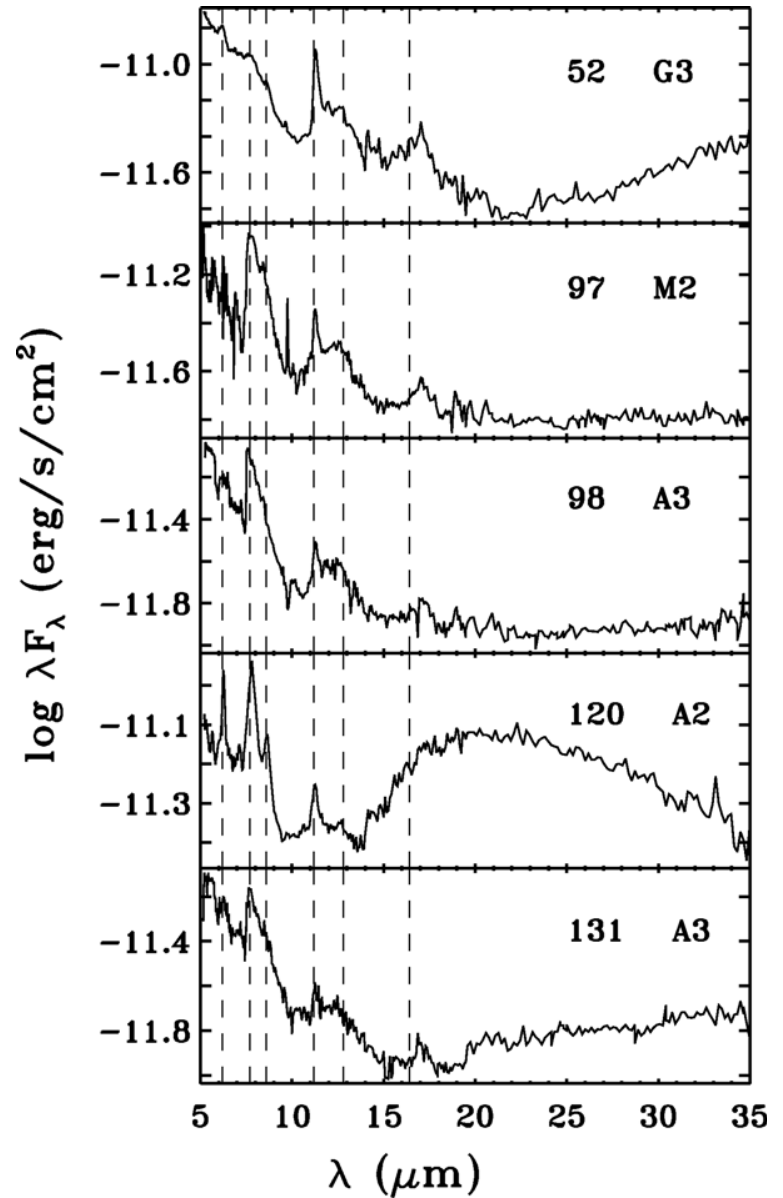

Figure 5. IRS spectra of the disk sources which show clear PAH features. The dashed lines indicate the positions of the most prominent bands (at 6.2, 7.7, 8.6, $11.2,12.8$, and $16.4 \mu \mathrm{m}$ ). The object number and its spectral type (from Oliveira et al. 2009) are indicated at the top right of each panel. Note that the ratios of different line strengths differ between sources.

extended nebulosity surrounding both positions so that the PAHs are likely associated with this nebula rather than the disks.

Discarding these two sources, this sample indicates that PAHs are present in $3.2 \%$ of the disk population studied, which is below the $11 \%-14 \%$ of PAH detection rate found by Geers (2007). This discrepancy could be due to the two samples being differently selected, with the current sample having a larger fraction of late-type stars. The percentage of PAHs present in disks in Serpens is, however, very comparable with that in Taurus (4\% of the disk population, Section 4.3).

Interestingly, none of the cold disks show PAHs, different from expected if grain settling enhances PAH features (Dullemond et al. 2007) and observed for other cold disk samples studied which do show PAHs (Brown et al. 2007; Merín et al. submitted). In addition, the objects with PAH emission have standard $F_{30} / F_{13}$ flux ratios, falling in the "flat" and 'flared' regions of Figure 3.

Only object 120 shows a silicate feature (at $20 \mu \mathrm{m}$, as discussed in Section 3.3.3), in addition to PAHs. This alludes to an anti-correlation between PAHs and silicates that could be simply explained in terms of contrast, i.e., it is hard to detect PAH features when a strong $10 \mu \mathrm{m}$ silicate feature is present (Geers et al. 2006). In this sense, PAHs should be more easily detected in disks without silicates, since the falling spectrum provides an increase in the feature to continuum ratio improving contrast.

\subsubsection{Silicate Emission}

Silicate emission has been observed around numerous circumstellar disks (e.g., van Boekel et al. 2003; Przygodda et al. 2003; Kessler-Silacci et al. 2006; Furlan et al. 2006; Bouwman et al. 2008; Pascucci et al. 2009; Olofsson et al. 2009). Some of this material has different characteristics in disks compared to primitive interstellar material. The strength and the shape of silicate features (at 10 and $20 \mu \mathrm{m}$ ) contain important information on the dust sizes in the surface layer of the disk. The 89 disk sources in this sample were inspected for their silicate characteristics. Their spectra can be seen in Figures 6-8 (completed with Figures 4 and 5), arranged in order of decreasing strength of the $10 \mu \mathrm{m}$ feature and decreasing $\alpha_{2-24 \mu \mathrm{m}}$ spectral slope. No extinction correction has been applied to these spectra (see Section 3.3.5).

Five of these sources (5\% of the disk population) have spectra that are featureless, i.e., they have neither the 10 nor the $20 \mu \mathrm{m}$ silicate features in emission, although they present IR excess (objects 38, 42, 59, 83, and 87). It is found that when the $10 \mu \mathrm{m}$ feature appears, the $20 \mu \mathrm{m}$ band is also present (by visual inspection of the continuum-subtracted spectra, see Section 3.3.4). The contrary is not true, as objects 13, 20, 24, $32,62,64,69,70,109,116,127,139,143$, and 145 have an observed $20 \mu \mathrm{m}$ feature but no apparent $10 \mu \mathrm{m}$ feature (also reported by Kessler-Silacci et al. (2006) for T Cha, SR21 and HD 135344B). Also, object 120 shows the $20 \mu \mathrm{m}$ band and no $10 \mu \mathrm{m}$ feature, as this region is dominated by PAH features in this source (see Section 3.3.2). When present, the $10 \mu \mathrm{m}$ feature $\left(S_{\text {peak }}^{10 \mu \mathrm{m}}\right)$ is usually stronger than the $20 \mu \mathrm{m}\left(S_{\text {peak }}^{20 \mu \mathrm{m}}\right)$ one as is common in disk sources.

Crystalline silicate features (as discussed by, e.g., Bouwman et al. 2001, 2008; Acke \& van den Ancker 2004; van Boekel et al. 2004; Apai et al. 2005; Olofsson et al. 2009; Sargent et al. 2009) are seen in several sources. A detailed analysis in terms of statistics and crystalline fraction of these features is non-trivial and beyond the scope of this paper, but will be the subject of a future publication.

\subsubsection{The Silicate Strength-Shape Relation}

As first shown for Herbig Ae stars (van Boekel et al. 2003), there is a dependence between grain size and silicate shape and strength for both 10 and $20 \mu \mathrm{m}$ features: as the sizes of the grains grow, the silicate features appear more and more flattened and shift to longer wavelengths.

A statistical analysis of these two features can be done using the following quantities: $S_{\text {peak }}^{10 \mu \mathrm{m}}$ is a measure of the strength of the feature centered at $10 \mu \mathrm{m}$ and $S_{11.3} / S_{9.8}$ is a proxy for the shape of the feature (the smaller this value, the more peaked the feature is); $S_{\text {peak }}^{20 \mu \mathrm{m}}$ measures the strength of the $20 \mu \mathrm{m}$ feature, while $S_{23.8} / S_{19}$ is a proxy for this feature shape. Following Kessler-Silacci et al. (2006), $S_{\lambda}$ is defined as

$$
S_{\lambda}=1+\frac{1}{2 \delta} \int_{\lambda-\delta}^{\lambda+\delta} \frac{F_{\lambda}-C_{\lambda}}{C_{\lambda}} d \lambda
$$

In this paper, we use $\delta=0.1 \mu \mathrm{m}$.

Kessler-Silacci et al. (2006) found a global trend (best fit to observations) for both features (Figure 9 in their paper and Figure 9 in this one), indicating that the feature shape and strength are physically related, as expected if the silicate features are indeed tracers of grain size. The relationship is much tighter 

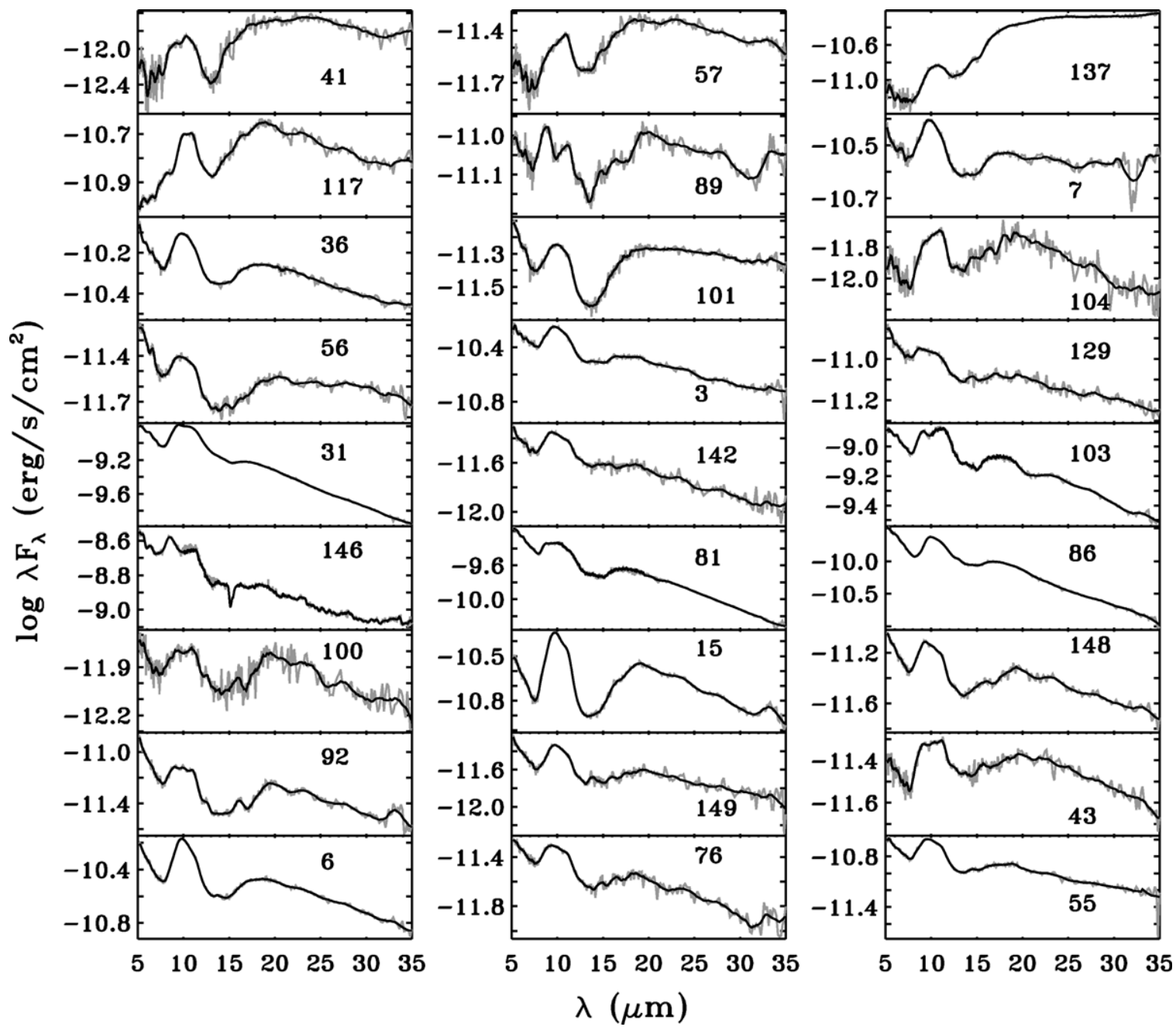

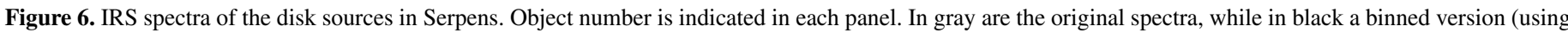
a Savitzky-Golay (Savitzky \& Golay 1964) filter of order 0 and degree 3) is overplotted.

for the $10 \mu \mathrm{m}$ feature, as also seen by the great spread in the correlation for the $20 \mu \mathrm{m}$ feature.

Following their procedure, we first subtracted the continuum of each spectrum by fitting a second-order polynomial to the following regions: blueward of the $10 \mu \mathrm{m}$ feature, between the 10 and the $20 \mu \mathrm{m}$ features, and redward of the $20 \mu \mathrm{m}$ feature $(6.8-7.5,12.5-13.5$ and $30-36 \mu \mathrm{m})$. The peak fluxes at $9.8,10$, $11.3,19,20$, and $23.8 \mu \mathrm{m}$ were then determined (see Table 2). Figure 9 shows the results for our sample of disks, excluding those with PAH emission. The best fit found by Kessler-Silacci et al. (2006) for the c2d IRS first look sample of YSOs has been indicated for comparison (solid line). The agreement of the Serpens data with the best fit of Kessler-Silacci et al. (2006) is excellent, and more evident for the $10 \mu \mathrm{m}$ than for the $20 \mu \mathrm{m}$ feature due to the larger scatter in the latter group.

Olofsson et al. (2009) generated synthetic $10 \mu \mathrm{m}$ features calculated for different grain sizes and compositions. Their models are generated for amorphous silicates of olivine and pyroxene stoichiometry and a 50:50 mixture, with grain size varying between 0.1 and $\sim 6 \mu \mathrm{m}$. Those models are overplotted in the left panel of Figure 9, with open symbols corresponding to different grain sizes. The comparison of the data presented here with these models shows that the majority of our sample lies in a region consistent with an opacity dominated by grains with sizes larger than $2.0 \mu \mathrm{m}$, having no features consistent with grain sizes smaller than $1.5 \mu \mathrm{m}$. A considerable fraction of the sample is consistent with grains as large as $6 \mu \mathrm{m}$ (the largest grain size modeled). The precise sizes depend on composition and treatment of the opacities, but sizes of a few $\mu \mathrm{m}$ are clearly implicated. It is important to note that crystallinity also affects the shape of silicate features. However, as shown by Apai et al. (2005) and Olofsson et al. (2009), the effect of crystallinity is orthogonal to that of grain sizes in the strength versus shape plot (Figure 9). Grain sizes are found to be the dominant parameter changing the shape of the $10 \mu \mathrm{m}$ silicate feature, whereas crystallinity introduces scatter (Figure 13 of Olofsson et al. 2009).

All cold disks present silicate features in emission. According to the models of grain sizes (the left panel of Figure 9), the cold disks in this sample have grains bigger than $2.7 \mu \mathrm{m}$, with the bulk of the sample presenting grains as big as $6.0 \mu \mathrm{m}$, according to these models.

\subsubsection{Effects of Extinction}

The amount of foreground extinction is, for some sources, substantial enough to affect the appearance of silicate emission features in the mid-infrared. Because the extinction law has strong resonances from silicates, the strength and shape of any silicate emission feature may be significantly affected if a large column of cloud material is present in front of a given source. 

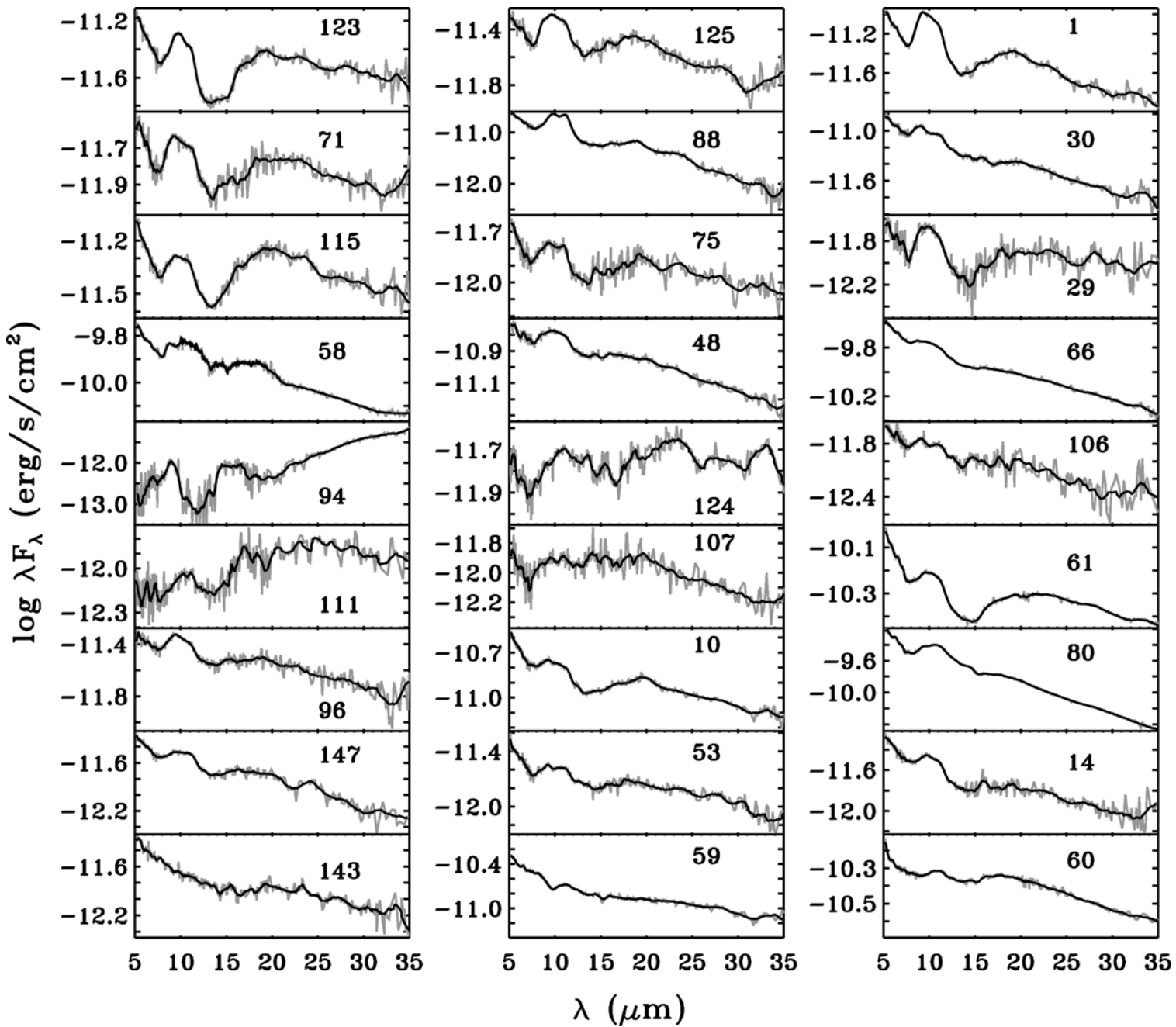

Figure 7. IRS spectra of the disk sources in Serpens, contd.

Here, the potential effects of extinction on statistical results, such as those presented in Figure 9, are discussed.

The model presented by Weingartner \& Draine (2001) for an absolute to selective extinction of $R_{V}=5.5$ has been found to be a reasonable match to the observed dark cloud extinction law (Chapman et al. 2009; McClure 2009; Chapman \& Mundy 2009), and it is assumed that this law holds for Serpens. One caveat is the lack of resonances due to ices in this model, but given other uncertainties in the determination of extinction laws, this is probably a minor contribution for the purposes of this work.

The opacity caused by a silicate resonance is defined as (e.g., Draine 2003)

$$
\Delta \tau_{\lambda}=\tau_{\lambda}-\tau_{C},
$$

where $\tau_{C}$ is the optical depth of the "continuum" opacity without the silicate resonance. The relation between optical extinction and $\Delta \tau$ is, using the fact that the optical depth is proportional to the extinction coefficient $C^{\text {ext }}$ :

$$
\frac{A_{V}}{\Delta \tau_{\lambda}}=\frac{\tau_{V}}{0.921 \Delta \tau_{\lambda}}=\frac{C_{V}^{\mathrm{ext}}}{0.921 \Delta C_{\lambda}^{\mathrm{ext}}} \equiv X_{\lambda} \text { mag. }
$$

Using Equations (1) and (3) and by approximating $\delta \rightarrow 0$, the silicate strength corrected for extinction is

$$
S_{\lambda}^{\mathrm{corr}} \sim \frac{S_{\lambda}}{\exp \left(-\Delta \tau_{\lambda}\right)}=\frac{S_{\lambda}}{\exp \left(-A_{V} / X_{\lambda}\right)} .
$$

For the $R_{V}=5.5$ extinction law of Weingartner \& Draine (2001), the relevant values for $X_{\lambda}$ are 19.6, 20.9, and $41.6 \mathrm{mag}$ for $9.8,10.0$, and $11.3 \mu \mathrm{m}$, respectively.

Using this relationship, it is possible to correct the position of sources with known values for $A_{V}$ in Figure 9. Oliveira et al. (2009) measured $A_{V}$ values for 49 of the 89 disks studied here albeit with some uncertainty. The resulting extinctioncorrected strength-shape distribution is shown in Figure 10 (in green) and compared with the uncorrected distribution (in black). It is seen that extinction moves points along curves that are almost parallel to the relation between shape and strength. Most points, having $A_{V}<5 \mathrm{mag}$, are not changed enough to alter the slope of the relation, and the median strength is unaffected. However, a few highly extincted disks are corrected by large amounts, as can be seen by the extinction arrows in Figure 10.

There are 40 YSOs in the sample for which Oliveira et al. (2009) do not estimate $A_{V}$. Some of these may be highly extincted $\left(A_{V} \gtrsim 10 \mathrm{mag}\right.$ ) sources (not being bright enough for optical spectroscopy using a $4 \mathrm{~m}$ class telescope). The correction for extinction, as derived above, would be greater for such objects, possibly introducing a displacement between the distributions of extinction corrected and uncorrected features. However, it is important to note that this assumes that the $R_{V}=5.5$ extinction law is valid for the densest regions of 

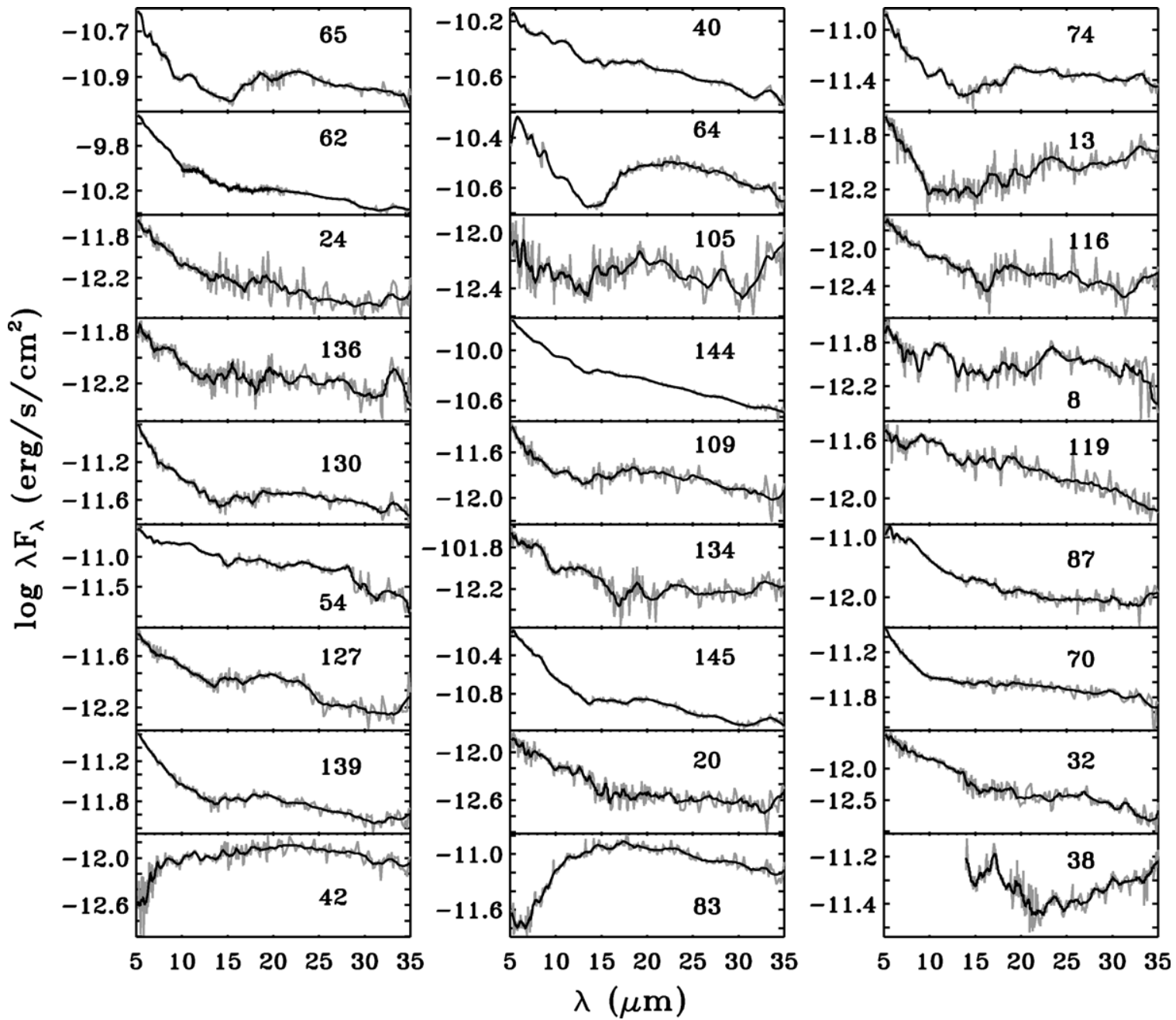

Figure 8. IRS spectra of the disk sources in Serpens, contd.
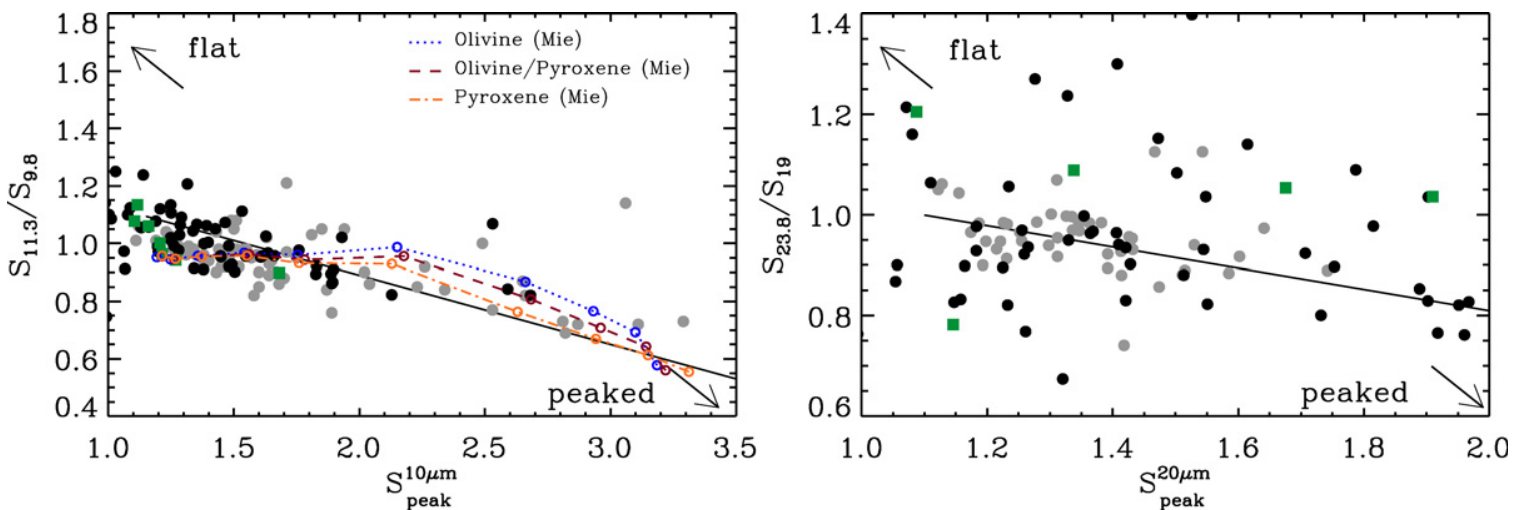

Figure 9. In the left panel, black dots show the ratio of peaks at 11.3 to $9.8 \mu \mathrm{m}\left(S_{11.3} / S_{9.8}\right)$ plotted against the peak at $10 \mu \mathrm{m}\left(S_{\text {peak }}^{10 \mu \mathrm{m}}\right)$. In the right panel, the ratio of peaks at 23.8 to $19 \mu \mathrm{m}\left(S_{23.8} / S_{19}\right)$ is plotted against the peak at $20 \mu \mathrm{m}\left(S_{\text {peak }}^{20 \mu \mathrm{m}}\right)$. The best fit found by Kessler-Silacci et al. (2006) for the c2d IRS first look sample of YSOs (gray dots) has been indicated for comparison (solid black line). Green squares are the cold disks in this sample (see Section 3.3.1). Colored curves are derived from theoretical opacities for different mixtures by Olofsson et al. (2009). The open circles correspond to different grain sizes, from left to right 6.25, 5.2, 4.3, 3.25, $2.7,2.0,1.5,1.25,1.0$, and $0.1 \mu \mathrm{m}$. Typical uncertainties for peak strengths are $\sim 0.1$, while typical uncertainties for the peak ratios are $\sim 0.12$.

(A color version of this figure is available in the online journal.)

molecular clouds. Chiar et al. (2007) recently found that at $A_{V}>10 \mathrm{mag}$, the relationship between optical depth and extinction derived for diffuse ISM is no longer valid, possibly due to grain growth, and that $X_{9.7 \mu \mathrm{m}}$ is significantly larger in this regime. If true, then the Weingartner \& Draine (2001) extinction law represents a "worst case" scenario. Since most of the measured extinctions are small, and given the uncertainty in extinction laws and the lack of $A_{V}$ data for a significant fraction of the sample, the uncorrected silicate feature strengths are used in the remainder of the paper. 


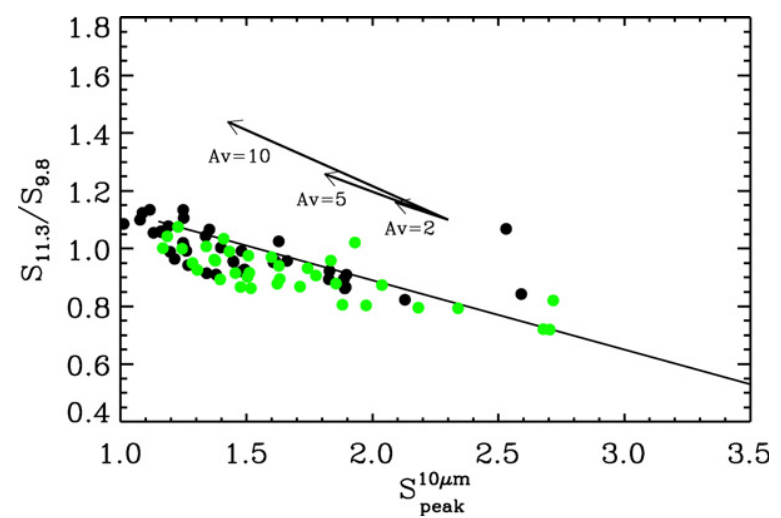

Figure 10. Comparison between the observed values (in black) and the extinction corrected values (in green) for the $10 \mu \mathrm{m}$ silicate feature. The arrows give an indication of the effects of extinction on the feature; however, their length depends on the starting point (see the text for details).

(A color version of this figure is available in the online journal.)

\subsubsection{Statistical Analysis of Silicate Features}

Figure 11 shows the strengths of the observed 10 and $20 \mu \mathrm{m}$ features with no apparent correlation. To quantify a correlation, a Kendall $\tau$ rank correlation coefficient is used to measure the degree of correspondence between two populations and to assess the significance of said correspondence. In other words, if there is a correlation (anti-correlation) between two data sets, the Kendall $\tau$ rank coefficient is equal to $1(-1)$. If the data sets are completely independent, the coefficient has value 0 . For the strengths of the 10 and $20 \mu \mathrm{m}$ silicate features, $\tau=0.26$, meaning at best a weak correlation.

The lack of apparent correlation between the 10 and the $20 \mu \mathrm{m}$ features, in both strength and appearance, suggests that they are emitted by different regions in the disk. Indeed, several authors have shown that the $10 \mu \mathrm{m}$ feature is emitted by warm dust in the inner region, while the $20 \mu \mathrm{m}$ feature originates from a colder component, further out and deeper into the disk (e.g., Kessler-Silacci et al. 2007; Merín et al. 2007; Bouwman et al. 2008; Olofsson et al. 2009). Therefore, the absence of the $10 \mu \mathrm{m}$ feature but presence of the $20 \mu \mathrm{m}$ band for a given source implies that such disk lacks warmer small dust grains close to the star. This is reminiscent of the disk around the Herbig Ae star HD 100453 studied by Meeus et al. (2002) and Vandenbussche et al. (2004), who find that the absence of the $10 \mu \mathrm{m}$ silicate feature can be fitted with a model deprived in warm small grains. 16\% of the disk sources shown in this sample fit this scenario. A possible explanation for such a scenario is that those disks have holes or gaps on such a small scale $(\lesssim 1 \mathrm{AU})$ that the holes do not produce a strong signature in the spectra probed by our data, as the cold disks do.

\section{DISCUSSION}

In this section we discuss the properties of the YSO sample presented in Sections 3.2 and 3.3 with respect to environment, and compare the results with those for another nearby starforming region, Taurus (Furlan et al. 2006), as well as the full c2d IRS sample (Kessler-Silacci et al. 2006; Olofsson et al. 2009).

\subsection{Cluster Versus Field Population}

Comparison of the disk properties between the cluster and field populations determines the importance of environment in

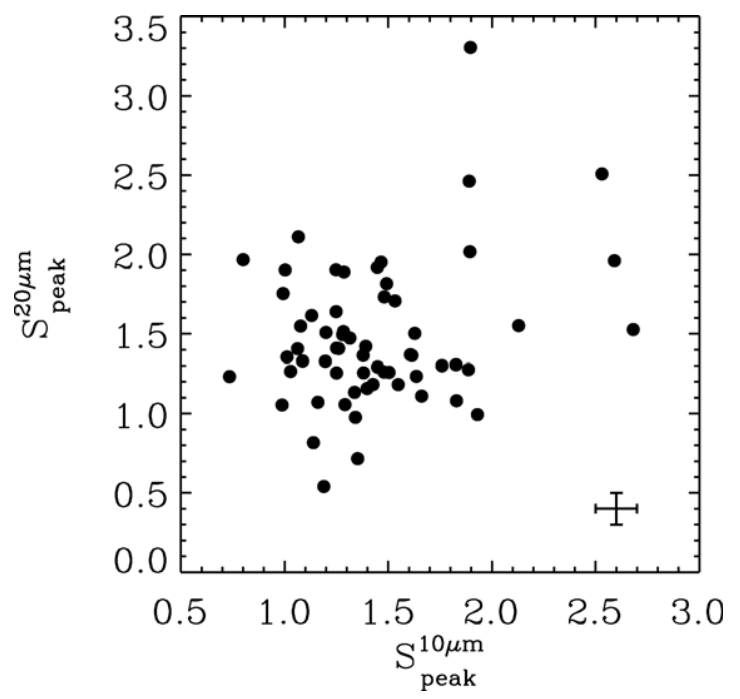

Figure 11. Relative strength of the $10 \mathrm{vs} .20 \mu \mathrm{m}$ silicate features. Typical error bars are shown in the bottom right corner. No obvious correlation is found.

the evolution of these systems. If the evolution of the disks in clusters is found to follow a different pace than that for more isolated stars, this can set important constraints on disk evolution theories.

As discussed in Harvey et al. (2006), the brightest YSOs in Serpens appear to be concentrated in clusters, but a more extended young stellar population exists outside these clusters. For the determination of clusters and their boundaries, we follow the method developed by Jørgensen et al. (2008) for Ophiuchus and Perseus. Using volume density and number of YSOs as criteria, the method consists of a nearest neighbor algorithm, and has as input the complete sample of YSOs in Serpens (Harvey et al. 2007b). The associations are divided into loose (volume density of $1 M_{\odot} \mathrm{pc}^{-3}$, blue contour in Figure 12) and tight (volume density of $25 M_{\odot} \mathrm{pc}^{-3}$, yellow contours in Figure 12). Furthermore, the associations are divided into clusters (more than 35 members) and groups (less than 35 members). The results of this method in Serpens yield 2 clusters (A and B) and 3 groups $(\mathrm{C}, \mathrm{D}$, and $\mathrm{E}$ ), that can be seen in Figure 12. Individual memberships are marked in Table 2. With the exception of one, virtually all YSOs in Serpens are within the blue contour, i.e., all YSOs belong to, at least, a loose association. This differs, for instance, from Ophiuchus and Perseus (Jørgensen et al. 2008), where some YSOs are completely isolated. For number statistics of clustering in all c2d clouds, see Evans et al. (2009).

Table 3 lists the number of objects in each tight association. It appears that the ratio of disk to embedded sources in a given cluster increases with distance from the densest part of the cluster, where Group C is located (see Figure 12).

To compare the populations, all objects belonging to any of the tight associations were grouped into the "cluster population," for better number statistics. Objects not belonging to any cluster were grouped into the "field population." Figure 13 shows the comparison between these two populations for three quantities derived from the IRS spectra. In the left panel, the flux ratio between 30 and $13 \mu \mathrm{m}\left(F_{30} / F_{13}\right)$ is an indication of disk geometry. The middle and right panels show the peak intensity of the silicate features at 10 and $20 \mu \mathrm{m}$, respectively. For all three quantities, the two populations (cluster and field) are statistically indistinguishable. A two sample Kolmogorov-Smirnov test (KS test) 


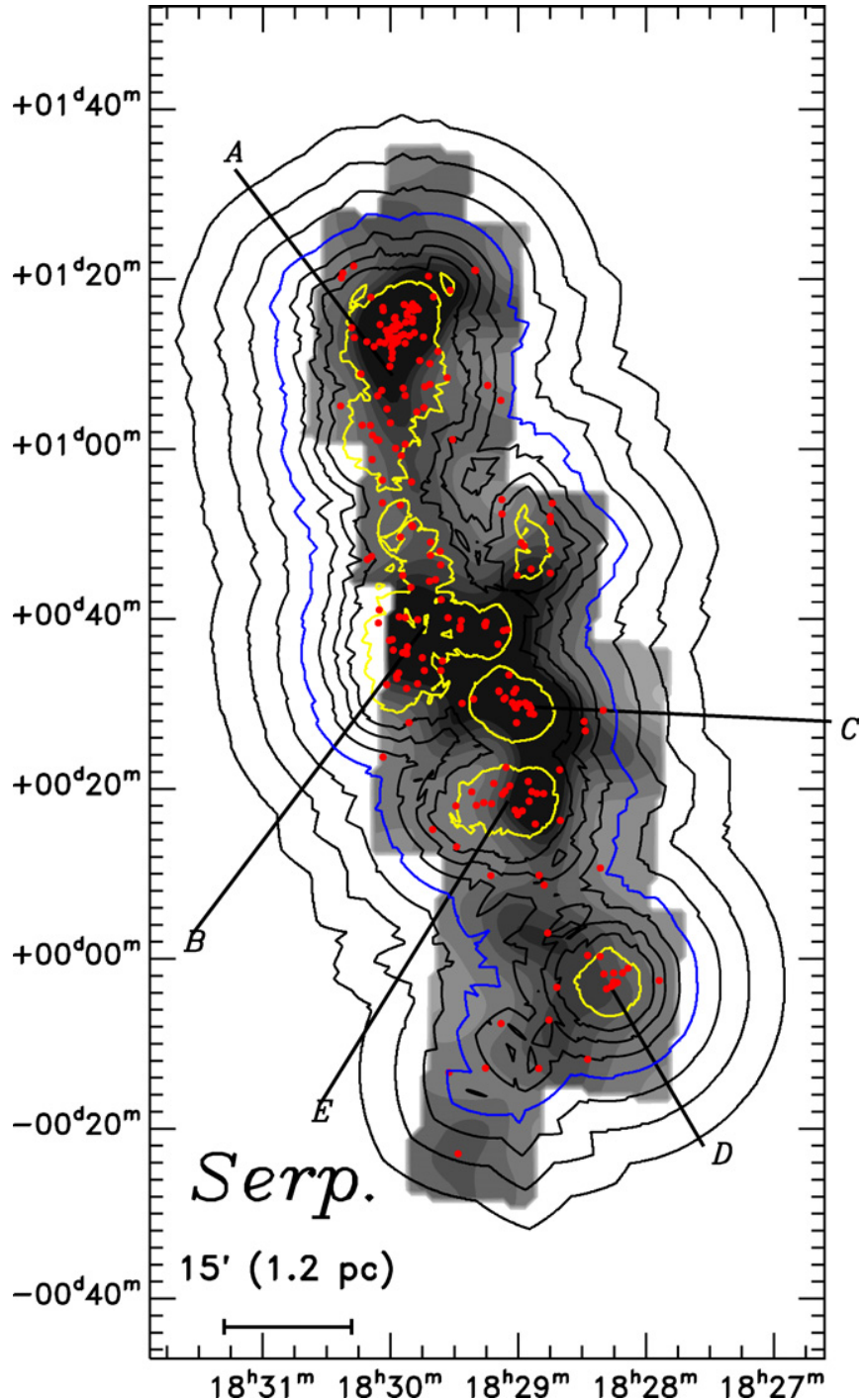

Figure 12. Clusters and groups in Serpens as defined according to the criteria in Jørgensen et al. (2008). The volume density contours are overlaid on the Serpens extinction map (Evans et al. 2007). The red dots are the YSOs in Serpens from Harvey et al. (2007b). Black contours indicate volume densities of 0.125, 0.25, $0.5,2.0$, and $4.0 M_{\odot} \mathrm{pc}^{-3}$, blue contour corresponds to volume density of $1 M_{\odot}$ $\mathrm{pc}^{-3}$ and yellow contours to a volume density of $25 M_{\odot} \mathrm{pc}^{-3}$.

(A color version of this figure is available in the online journal.)

was performed for each quantity and the results show that the null hypothesis that the two distributions come from the same parent population cannot be rejected to any significance (18\%, $13 \%$, and $79 \%$ for $F_{30} / F_{13}, S_{\text {peak }}^{10 \mu \mathrm{m}}$, and $S_{\text {peak }}^{20 \mu \mathrm{m}}$, respectively).

Differences between the cluster versus field populations will be further investigated with ancillary data (e.g., relative stellar ages and masses) and modeling (e.g., disk sizes). However, the IRS spectra allow the conclusion that no significant differences are found for disk geometry or the grain size distribution in the upper layers of circumstellar disks in clustered compared with field stars. The latter result only applies to the inner disk, as traced by silicate features. The outer disk may still be different between cluster and field populations.

\subsection{Comparison with Other Samples}

The young stars with disks observed by the c2d team with IRS spectroscopy (c2d IRS sample) are scattered across the sky in the five molecular clouds studied (Olofsson et al. 2009). The other four clouds studied by the $\mathrm{c} 2 \mathrm{~d}$ are Chamaeleon II, Lupus, Perseus, and Ophiuchus. All clouds are nearby (within 300 pc), span a range of star formation activity, have typical median ages of a few Myr and have a spread between more (Perseus and Ophiuchus) and less (Cha II and Lupus) clustered YSO populations (Evans et al. 2009). The results from this sample are also compared to the results in Serpens in Figures 9 and 13. A conspicuous similarity is seen between the samples. Note that Olofsson et al. (2009) do not analyze the $20 \mu \mathrm{m}$ silicate feature in this same manner and, therefore, $S_{\text {peak }}^{20 \mu \mathrm{m}}$ for the c2d IRS sample is missing from the right panel in Figure 13.

The models of Dullemond \& Dominik (2008) conclude that, if sedimentation is the unique reason for the variety of observed strength and shape of the $10 \mu \mathrm{m}$ silicate feature, then this feature is strong for weak excess in mid- to far-IR (as probed by $\left.F_{30} / F_{13}\right)$, and vice versa. However, when studying a small sample of T Tauri stars from the Spitzer Legacy Program "The Formation and Evolution of Planetary Systems: Placing Our Solar System in Context" (FEPS) sample, Bouwman et al. (2008) found the opposite: a trend in which weak $F_{30} / F_{13}$ correlates with a weak feature. A confirmation of this trend for a larger sample implies that sedimentation alone cannot be the sole cause for the diversity of observed silicate features. Dullemond \& Dominik (2008) argue that dust coagulation must then play a vital role in producing different silicate profiles. In Figure 14, we populate this diagram with the Serpens sample (black dots) and the c2d IRS sample (blue stars), as well as the Bouwman FEPS sample (red triangles). This large combined sample shows no correlation between the strength of the $10 \mu \mathrm{m}$
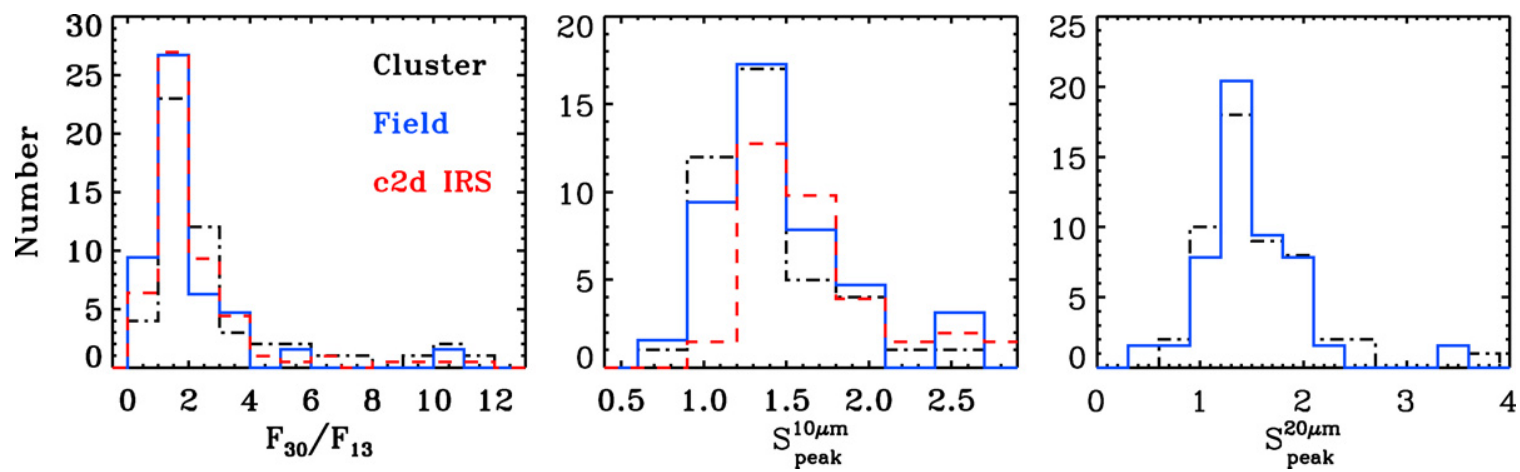

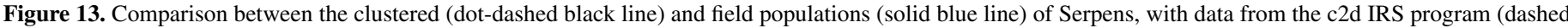

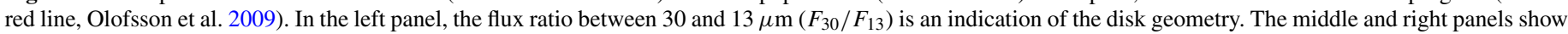
the strength of the silicate features at 10 and $20 \mu \mathrm{m}$, respectively.

(A color version of this figure is available in the online journal.) 
Table 2

Characteristics of YSOs in Serpens

\begin{tabular}{|c|c|c|c|c|c|c|c|c|c|c|}
\hline No. & Emb & $\mathrm{PAH}^{\mathrm{a}}$ & $S_{9.8}$ & $S_{11.3}$ & $S_{\text {peak }}^{10 \mu \mathrm{m}}$ & $S_{19.0}$ & $S_{23.8}$ & $S_{\text {peak }}^{20 \mu \mathrm{m}}$ & Cluster & Spectral Type \\
\hline 1 & $\ldots$ & $\ldots$ & 2.53 & 2.13 & 2.59 & 2.00 & 1.52 & 1.96 & & $\mathrm{~K} 2$ \\
\hline 3 & $\ldots$ & $\ldots$ & 1.49 & 1.35 & 1.50 & 1.33 & 1.23 & 1.26 & $\mathrm{D}$ & M0 \\
\hline 6 & $\ldots$ & $\ldots$ & 2.12 & 1.73 & 2.13 & 1.61 & 1.33 & 1.55 & D & K5 \\
\hline 7 & $\ldots$ & $\ldots$ & 1.38 & 1.25 & 1.38 & 1.39 & 1.34 & 1.36 & D & M0 \\
\hline 8 & $\ldots$ & $\ldots$ & 1.15 & 1.43 & 1.14 & 1.21 & 1.45 & 0.82 & $\mathrm{D}$ & \\
\hline 9 & $\ldots$ & $\ldots$ & 1.64 & 1.48 & 1.68 & 2.24 & 1.97 & 2.27 & $\mathrm{D}$ & \\
\hline 10 & $\ldots$ & $\ldots$ & 1.25 & 1.29 & 1.28 & 1.47 & 1.29 & 1.51 & $\mathrm{D}$ & \\
\hline 13 & $\ldots$ & $\ldots$ & $\ldots$ & $\ldots$ & $\ldots$ & 1.33 & 1.87 & 1.29 & $\mathrm{D}$ & \\
\hline 14 & $\ldots$ & $\ldots$ & 1.45 & 1.38 & 1.45 & 1.65 & 1.43 & 1.53 & & M2 \\
\hline 15 & $\ldots$ & $\ldots$ & 2.67 & 2.19 & 2.68 & 2.59 & 2.07 & 2.46 & $\mathrm{D}$ & \\
\hline 20 & $\ldots$ & $\ldots$ & $\ldots$ & $\ldots$ & $\ldots$ & 0.90 & 0.85 & 1.13 & & \\
\hline 21 & $\ldots$ & $\ldots$ & 1.09 & 1.17 & 1.10 & 1.45 & 1.57 & 1.34 & & \\
\hline 24 & $\ldots$ & $\ldots$ & $\ldots$ & $\ldots$ & $\ldots$ & 1.69 & 1.29 & 1.30 & & \\
\hline 26 & $\mathrm{Y}$ & $\ldots$ & $\ldots$ & $\ldots$ & $\ldots$ & $\ldots$ & $\ldots$ & $\ldots$ & & \\
\hline 27 & $\mathrm{Y}$ & $\ldots$ & $\ldots$ & $\ldots$ & $\ldots$ & $\ldots$ & $\ldots$ & $\ldots$ & & \\
\hline 28 & $\mathrm{Y}$ & $\ldots$ & $\ldots$ & $\ldots$ & $\ldots$ & $\ldots$ & $\ldots$ & $\ldots$ & & \\
\hline 29 & $\ldots$ & $\ldots$ & 1.84 & 1.59 & 1.89 & 1.56 & 2.03 & 1.73 & & M2 \\
\hline 30 & $\ldots$ & $\ldots$ & 1.32 & 1.38 & 1.34 & 1.22 & 1.18 & 1.33 & & M1 \\
\hline 31 & $\ldots$ & $\ldots$ & 1.76 & 1.72 & 1.76 & 0.99 & 0.92 & 0.99 & & \\
\hline 32 & $\ldots$ & $\ldots$ & $\ldots$ & $\ldots$ & $\ldots$ & 0.89 & 1.13 & 0.72 & & \\
\hline 36 & $\ldots$ & $\ldots$ & 1.47 & 1.35 & 1.48 & 1.41 & 1.31 & 1.41 & & K5 \\
\hline 38 & $\ldots$ & $\ldots$ & $\ldots$ & $\ldots$ & $\ldots$ & $\ldots$ & $\ldots$ & $\ldots$ & & \\
\hline 39 & $\mathrm{Y}$ & $\ldots$ & $\ldots$ & $\ldots$ & $\ldots$ & $\ldots$ & $\ldots$ & $\ldots$ & $\mathrm{E}$ & \\
\hline 40 & $\ldots$ & $\ldots$ & 1.06 & 1.20 & 1.09 & 1.29 & 1.26 & 1.25 & $\mathrm{E}$ & M7 \\
\hline 41 & $\ldots$ & $\ldots$ & 1.73 & 1.77 & 1.93 & 3.45 & 4.18 & 3.74 & $\mathrm{C}$ & $\mathrm{K} 2$ \\
\hline 42 & $\ldots$ & $\ldots$ & $\ldots$ & $\ldots$ & $\ldots$ & $\ldots$ & $\ldots$ & $\ldots$ & $\mathrm{E}$ & \\
\hline 43 & $\ldots$ & $\ldots$ & 1.32 & 1.40 & 1.35 & 1.18 & 1.18 & 1.28 & & M0.5 \\
\hline 44 & $\mathrm{Y}$ & $\ldots$ & $\ldots$ & $\ldots$ & $\ldots$ & $\ldots$ & $\ldots$ & $\ldots$ & $\mathrm{C}$ & M6 \\
\hline 45 & $\mathrm{Y}$ & $\ldots$ & $\ldots$ & $\ldots$ & $\ldots$ & $\ldots$ & $\ldots$ & $\ldots$ & $\mathrm{C}$ & \\
\hline 47 & $\mathrm{Y}$ & $\ldots$ & $\ldots$ & $\ldots$ & $\ldots$ & $\ldots$ & $\ldots$ & $\ldots$ & $\mathrm{E}$ & M5 \\
\hline 48 & $\ldots$ & $\ldots$ & 1.22 & 1.21 & 1.26 & 1.12 & 1.05 & 1.18 & $\mathrm{E}$ & M5.5 \\
\hline 49 & $\mathrm{Y}$ & $\ldots$ & $\ldots$ & $\ldots$ & $\ldots$ & $\ldots$ & $\ldots$ & $\ldots$ & $\mathrm{C}$ & \\
\hline 50 & $\mathrm{Y}$ & $\ldots$ & $\ldots$ & $\ldots$ & $\ldots$ & $\ldots$ & $\ldots$ & $\ldots$ & $\mathrm{C}$ & \\
\hline 51 & $\mathrm{Y}$ & $\ldots$ & $\ldots$ & $\ldots$ & $\ldots$ & $\ldots$ & $\ldots$ & $\ldots$ & & \\
\hline 52 & $\ldots$ & $\mathrm{Y}$ & $\ldots$ & $\ldots$ & $\ldots$ & $\ldots$ & $\ldots$ & $\ldots$ & $\mathrm{E}$ & G3 \\
\hline 53 & $\ldots$ & $\ldots$ & 1.19 & 1.32 & 1.25 & 1.26 & 1.04 & 1.18 & & M2.5 \\
\hline 54 & $\ldots$ & $\ldots$ & 1.13 & 1.26 & 1.21 & 1.19 & 1.47 & 1.07 & $\mathrm{C}$ & \\
\hline 55 & $\ldots$ & $\ldots$ & 1.88 & 1.68 & 1.89 & 1.53 & 1.18 & 1.35 & $\mathrm{E}$ & $\mathrm{K} 2$ \\
\hline 56 & $\ldots$ & $\ldots$ & 1.56 & 1.49 & 1.55 & 1.32 & 1.23 & 1.41 & & \\
\hline 57 & $\ldots$ & $\ldots$ & 1.39 & 1.47 & 1.43 & 2.01 & 1.81 & 1.90 & $\mathrm{C}$ & \\
\hline 58 & $\ldots$ & $\ldots$ & 1.09 & 1.15 & 1.16 & 1.13 & 1.20 & 1.33 & $\mathrm{C}$ & K7 \\
\hline 59 & $\ldots$ & $\ldots$ & $\ldots$ & $\ldots$ & $\ldots$ & $\ldots$ & $\ldots$ & $\ldots$ & $\mathrm{C}$ & \\
\hline 60 & $\ldots$ & $\ldots$ & 1.00 & 1.08 & 1.01 & 1.27 & 1.25 & 1.26 & $\mathrm{C}$ & M0.5 \\
\hline 61 & $\ldots$ & $\ldots$ & 1.24 & 1.25 & 1.25 & 1.28 & 1.32 & 1.26 & $\mathrm{C}$ & M0 \\
\hline 62 & $\ldots$ & $\ldots$ & $\ldots$ & $\ldots$ & $\ldots$ & 1.21 & 1.31 & 1.26 & $\mathrm{C}$ & K0 \\
\hline 63 & $\mathrm{Y}$ & $\ldots$ & $\ldots$ & $\ldots$ & $\ldots$ & $\ldots$ & $\ldots$ & $\ldots$ & $\mathrm{C}$ & \\
\hline 64 & $\ldots$ & $\ldots$ & $\ldots$ & $\ldots$ & $\ldots$ & 1.41 & 1.27 & 1.36 & $\mathrm{C}$ & \\
\hline 65 & $\ldots$ & $\ldots$ & 1.00 & 1.10 & 1.00 & 1.21 & 1.12 & 1.06 & $\mathrm{C}$ & \\
\hline 66 & $\ldots$ & $\ldots$ & 1.19 & 1.18 & 1.20 & 1.15 & 1.07 & 1.11 & $\mathrm{E}$ & K5 \\
\hline 67 & $\mathrm{Y}$ & $\ldots$ & $\ldots$ & $\ldots$ & $\ldots$ & $\ldots$ & $\ldots$ & $\ldots$ & $\mathrm{C}$ & \\
\hline 69 & $\ldots$ & $\ldots$ & $\ldots$ & $\ldots$ & $\ldots$ & 2.14 & 2.17 & 2.27 & B & \\
\hline 70 & $\ldots$ & $\ldots$ & $\ldots$ & $\ldots$ & $\ldots$ & 1.29 & 1.36 & 1.31 & $\mathrm{E}$ & $\mathrm{A} 3$ \\
\hline 71 & $\ldots$ & $\ldots$ & 1.50 & 1.49 & 1.48 & 2.48 & 2.86 & 2.51 & $\mathrm{E}$ & M3 \\
\hline 72 & $\mathrm{Y}$ & $\ldots$ & $\ldots$ & $\ldots$ & $\ldots$ & $\ldots$ & $\ldots$ & $\ldots$ & $\mathrm{C}$ & \\
\hline 73 & $\mathrm{Y}$ & $\ldots$ & $\ldots$ & $\ldots$ & $\ldots$ & $\ldots$ & $\ldots$ & $\ldots$ & $\mathrm{C}$ & \\
\hline 74 & $\ldots$ & $\ldots$ & 0.99 & 1.24 & 1.03 & 1.44 & 1.33 & 1.55 & B & \\
\hline 75 & $\ldots$ & $\ldots$ & 1.37 & 1.37 & 1.38 & 1.64 & 1.35 & 1.50 & & \\
\hline 76 & $\ldots$ & $\ldots$ & 1.58 & 1.51 & 1.61 & 1.83 & 1.78 & 1.75 & & M1 \\
\hline 79 & $\mathrm{Y}$ & $\ldots$ & $\ldots$ & $\ldots$ & $\ldots$ & $\ldots$ & $\ldots$ & $\ldots$ & $\mathrm{C}$ & \\
\hline 80 & $\ldots$ & $\ldots$ & 1.27 & 1.39 & 1.29 & 0.96 & 0.92 & 0.98 & B & \\
\hline 81 & $\ldots$ & $\ldots$ & 1.59 & 1.52 & 1.66 & 1.50 & 1.28 & 1.42 & & M5 \\
\hline 82 & $\ldots$ & $\ldots$ & 1.31 & 1.23 & 1.27 & 1.14 & 0.90 & 1.15 & $\mathrm{E}$ & M0 \\
\hline 83 & $\ldots$ & $\ldots$ & $\ldots$ & $\ldots$ & $\ldots$ & $\ldots$ & $\ldots$ & $\ldots$ & $\mathrm{E}$ & \\
\hline 86 & $\ldots$ & $\ldots$ & 1.80 & 1.61 & 1.83 & 1.28 & 0.98 & 1.23 & & M5.5 \\
\hline 87 & $\ldots$ & $\ldots$ & $\ldots$ & $\ldots$ & $\ldots$ & $\ldots$ & $\ldots$ & $\ldots$ & B & M4 \\
\hline 88 & $\ldots$ & $\ldots$ & 2.58 & 2.75 & 2.53 & 1.70 & 1.41 & 1.47 & & M0.5 \\
\hline
\end{tabular}


Table 2

(Continued)

\begin{tabular}{|c|c|c|c|c|c|c|c|c|c|c|}
\hline No. & Emb & $\mathrm{PAH}^{\mathrm{a}}$ & $S_{9.8}$ & $S_{11.3}$ & $S_{\text {peak }}^{10 \mu \mathrm{m}}$ & $S_{19.0}$ & $S_{23.8}$ & $S_{\text {peak }}^{20 \mu \mathrm{m}}$ & Cluster & Spectral Type \\
\hline 89 & $\ldots$ & $\ldots$ & 1.10 & 1.21 & 1.08 & 1.62 & 1.68 & 1.71 & B & K5 \\
\hline 90 & $\ldots$ & $\ldots$ & 1.19 & 1.19 & 1.21 & 1.54 & 1.62 & 1.67 & B & \\
\hline 91 & $\mathrm{Y}$ & $\ldots$ & $\ldots$ & $\ldots$ & $\ldots$ & $\ldots$ & $\ldots$ & $\ldots$ & E & K7 \\
\hline 92 & $\ldots$ & $\ldots$ & 1.56 & 1.60 & 1.63 & 1.92 & 1.66 & 1.97 & E & M0 \\
\hline 94 & $\ldots$ & $\ldots$ & 1.14 & 0.85 & 0.99 & 0.46 & 0.53 & 0.54 & & \\
\hline 96 & $\ldots$ & $\ldots$ & 1.35 & 1.23 & 1.34 & 1.53 & 1.48 & 1.41 & E & M1 \\
\hline 97 & $\ldots$ & $\mathrm{Y}$ & $\ldots$ & $\ldots$ & $\ldots$ & $\ldots$ & $\ldots$ & $\ldots$ & & M2 \\
\hline 98 & $\ldots$ & $\mathrm{Y}$ & $\ldots$ & $\ldots$ & $\ldots$ & $\ldots$ & $\ldots$ & $\ldots$ & & $\mathrm{A} 3$ \\
\hline 100 & $\ldots$ & $\ldots$ & 1.39 & 1.47 & 1.39 & 1.90 & 1.56 & 1.89 & & \\
\hline 101 & $\ldots$ & $\ldots$ & 1.60 & 1.55 & 1.63 & 1.95 & 1.65 & 1.92 & B & \\
\hline 102 & $\mathrm{Y}$ & $\ldots$ & $\ldots$ & $\ldots$ & $\ldots$ & $\ldots$ & $\ldots$ & $\ldots$ & B & \\
\hline 103 & $\ldots$ & $\ldots$ & 1.27 & 1.53 & 1.31 & 1.30 & 1.28 & 1.16 & & \\
\hline 104 & $\ldots$ & $\ldots$ & 1.50 & 1.67 & 1.53 & 1.96 & 1.70 & 1.90 & & \\
\hline 105 & $\ldots$ & $\ldots$ & 0.87 & 0.87 & 0.80 & 3.84 & 3.15 & 3.30 & & \\
\hline 106 & $\ldots$ & $\ldots$ & 1.19 & 1.28 & 1.19 & 1.22 & 1.42 & 1.61 & & M3 \\
\hline 107 & $\ldots$ & $\ldots$ & 1.07 & 1.04 & 1.06 & 1.24 & 1.16 & 1.37 & & \\
\hline 109 & $\ldots$ & $\ldots$ & $\ldots$ & $\ldots$ & $\ldots$ & 1.58 & 1.31 & 1.51 & B & \\
\hline 111 & $\ldots$ & $\ldots$ & 1.24 & 1.32 & 1.29 & 0.61 & 1.11 & 1.23 & & \\
\hline 113 & $\cdots$ & $\cdots$ & 1.10 & 1.25 & 1.12 & 1.76 & 1.82 & 1.91 & & K7 \\
\hline 114 & $\ldots$ & $\ldots$ & 1.14 & 1.21 & 1.16 & 1.90 & 1.99 & 2.05 & B & F9 \\
\hline 115 & $\ldots$ & $\ldots$ & 1.43 & 1.36 & 1.45 & 2.22 & 2.06 & 2.11 & & M0.5 \\
\hline 116 & $\ldots$ & $\ldots$ & $\ldots$ & $\ldots$ & $\ldots$ & 1.56 & 1.51 & 1.49 & B & \\
\hline 117 & $\ldots$ & $\ldots$ & 1.37 & 1.38 & 1.40 & 1.87 & 1.68 & 1.81 & & $\mathrm{~K} 2$ \\
\hline 119 & $\ldots$ & $\ldots$ & 1.20 & 1.23 & 1.25 & 1.22 & 0.82 & 1.05 & B & K7 \\
\hline 120 & $\ldots$ & $\mathrm{Y}$ & $\ldots$ & $\ldots$ & $\ldots$ & 1.70 & 1.52 & 1.64 & B & A2 \\
\hline 122 & $\ldots$ & $\ldots$ & 1.22 & 1.17 & 1.21 & 0.90 & 1.08 & 1.09 & B & M0 \\
\hline 123 & $\ldots$ & $\ldots$ & 1.87 & 1.70 & 1.90 & 1.97 & 1.63 & 1.95 & B & M0 \\
\hline 124 & $\ldots$ & $\ldots$ & 1.09 & 1.15 & 1.13 & 1.06 & 1.15 & 1.08 & B & M0 \\
\hline 125 & $\ldots$ & $\ldots$ & 1.57 & 1.50 & 1.61 & 2.06 & 1.86 & 2.02 & B & M0 \\
\hline 127 & $\ldots$ & $\ldots$ & $\ldots$ & $\ldots$ & $\ldots$ & 1.85 & 1.71 & 2.00 & B & M2 \\
\hline 129 & $\cdots$ & $\ldots$ & 1.19 & 1.16 & 1.20 & 1.11 & 0.95 & 1.15 & & \\
\hline 130 & $\ldots$ & $\ldots$ & $\ldots$ & $\ldots$ & $\ldots$ & 1.39 & 1.24 & 1.23 & & K6 \\
\hline 131 & $\ldots$ & $\mathrm{Y}$ & $\ldots$ & $\ldots$ & $\ldots$ & $\ldots$ & $\ldots$ & $\ldots$ & & $\mathrm{A} 3$ \\
\hline 132 & $\mathrm{Y}$ & $\ldots$ & $\ldots$ & $\ldots$ & $\ldots$ & $\ldots$ & $\ldots$ & $\ldots$ & B & \\
\hline 133 & $\mathrm{Y}$ & $\ldots$ & $\ldots$ & $\ldots$ & $\ldots$ & $\ldots$ & $\ldots$ & $\ldots$ & B & \\
\hline 134 & $\ldots$ & $\ldots$ & 0.76 & 0.88 & 0.73 & 1.25 & 1.33 & 0.89 & B & \\
\hline 135 & $\mathrm{Y}$ & $\ldots$ & $\ldots$ & $\ldots$ & $\ldots$ & $\ldots$ & $\ldots$ & $\ldots$ & B & \\
\hline 136 & $\ldots$ & $\ldots$ & 1.08 & 0.98 & 1.07 & 1.30 & 1.38 & 1.54 & B & \\
\hline 137 & $\ldots$ & $\ldots$ & 1.27 & 1.24 & 1.28 & 1.99 & 2.06 & 2.01 & B & \\
\hline 139 & $\ldots$ & $\ldots$ & $\ldots$ & $\ldots$ & $\ldots$ & 1.88 & 1.50 & 1.76 & & $\mathrm{~A} 4$ \\
\hline 140 & $\mathrm{Y}$ & $\ldots$ & $\ldots$ & $\ldots$ & $\ldots$ & $\ldots$ & $\ldots$ & $\ldots$ & B & \\
\hline 142 & $\ldots$ & $\ldots$ & 1.50 & 1.39 & 1.49 & 1.26 & 1.13 & 1.16 & B & M4 \\
\hline 143 & $\ldots$ & $\cdots$ & $\ldots$ & $\ldots$ & $\ldots$ & 1.42 & 1.26 & 1.47 & B & \\
\hline 144 & $\ldots$ & $\ldots$ & 0.98 & 1.11 & 0.99 & 1.30 & 1.23 & 1.32 & B & \\
\hline 145 & $\ldots$ & $\ldots$ & $\ldots$ & $\ldots$ & $\ldots$ & 1.27 & 1.26 & 1.32 & B & G2.5 \\
\hline 146 & $\ldots$ & $\ldots$ & 1.23 & 1.39 & 1.25 & 1.28 & 1.15 & 1.22 & A & M4 \\
\hline 147 & $\ldots$ & $\ldots$ & 1.41 & 1.51 & 1.46 & 1.40 & 1.23 & 1.42 & B & \\
\hline 148 & $\ldots$ & $\ldots$ & 1.81 & 1.67 & 1.83 & 1.76 & 1.51 & 1.79 & B & K7 \\
\hline 149 & $\ldots$ & $\ldots$ & 1.90 & 1.65 & 1.89 & 1.47 & 1.31 & 1.43 & & M0 \\
\hline
\end{tabular}

Note. ${ }^{a}$ All disk sources with PAH in emission show the features at 6.2, 7.7, 8.6, 11.2, and $12.8 \mu \mathrm{m}$.

silicate feature and $F_{30} / F_{13}(\tau=0.07)$ and therefore does not support either the correlation (as seen by Bouwman et al. 2008) or the anti-correlation (as modeled by Dullemond \& Dominik 2008 for sedimentation alone) between the IR flux excess and the strength of the $10 \mu \mathrm{m}$ silicate feature.

\subsection{Comparison with Taurus}

The Taurus Molecular Cloud is the best characterized starforming region to date, due to its proximity and relatively low extinction. With young stars and their surrounding disks studied for more than two decades (e.g., Kenyon \& Hartmann 1987, 1995), its members have been well characterized at a wide range of wavelengths. Taurus has thus become the reference for comparison of star-forming regions. Compared to Serpens, Taurus seems to be a somewhat younger (2.0 Myr median age, Hartmann et al. 2001 versus 4.7 Myr Oliveira et al. 2009) and has a lower star-forming rate. Due to uncertainties in premain sequence age determinations (e.g., Baraffe et al. 2009; Hillenbrand 2009; Naylor 2009), this difference may not be significant. However, cluster ages are statistically more likely to be different than the same.

In a campaign similar to that presented here, IRS spectra were obtained for a sample of 139 YSOs in Taurus, as part of a larger IRS guaranteed-time observing program. These data were 


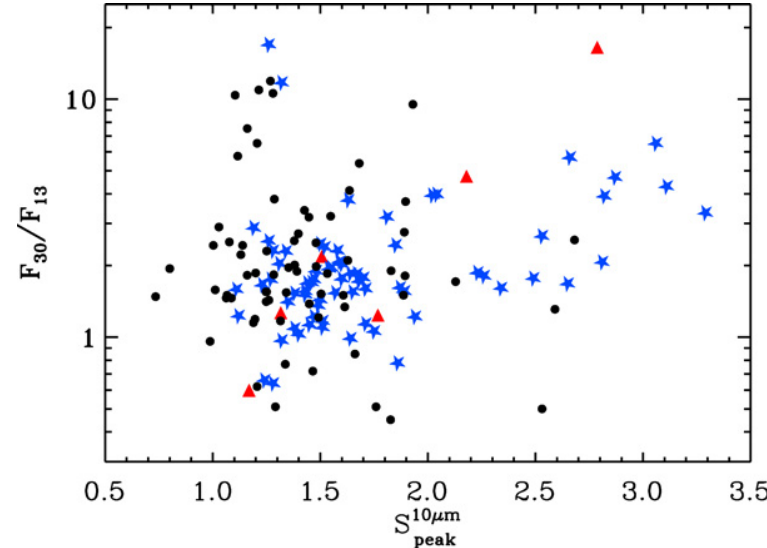

Figure 14. Flux ratio between 30 and $13 \mu \mathrm{m}\left(F_{30} / F_{13}\right)$ plotted against the peak at $10 \mu \mathrm{m}$ ( $S_{\mathrm{peak}}^{10 \mu \mathrm{m}}$, black dots), compared against the T Tauri FEPS sample (red triangles; Bouwman et al. 2008) and the c2d IRS sample (blue stars; Olofsson et al. 2009). Typical uncertainties for the $10 \mu \mathrm{m}$ peak strength are $\sim 0.1$, while typical uncertainties for the flux ratio are $\sim 0.12$.

(A color version of this figure is available in the online journal.)

Table 3

Number of Objects in Tight Groups and Associations

\begin{tabular}{cccc}
\hline \hline Group & Embedded & Disks & Total \\
\hline Cluster A & 0 & 1 & $1^{\mathrm{a}}$ \\
Cluster B & 5 & 26 & 31 \\
Group C & 9 & 10 & 19 \\
Group D & 0 & 8 & 8 \\
Group E & 3 & 12 & 15
\end{tabular}

Note. ${ }^{\mathrm{a}}$ The Serpens Core, or Cluster A, is not complete in these observations.

presented in two separate papers: Furlan et al. (2006) treated the disk sources, while Furlan et al. (2008) presented the embedded population in Taurus.

Because the young stellar population in Serpens has been discovered and characterized using IR observations, this sample does not include young stars without disks (and therefore no IR excess, also called Class III sources, as defined in Lada 1987). For this reason, a comparison between the Class III sources of the two clouds does not make sense. Twenty-six Class III objects were studied in Taurus. These objects present featureless spectra with very little IR excess at longer wavelengths. It is, however, possible to compare the IR excess populations of both regions. Out of the entire YSO sample studied in Taurus, embedded sources (or Class I) amount to $20 \%$ while in Serpens they amount to $18 \%$, a very comparable number. This also matches the typical percentage of Class I sources in the global YSO statistics of the five clouds from the c2d photometric sample (Evans et al. 2009). In Serpens only five objects have featureless spectra.

Excluding the embedded sources to analyze the disk population alone, objects having both 10 and $20 \mu \mathrm{m}$ silicate features amount to $72 \%$ in Taurus, comparable to $73 \%$ of the Serpens disk population. Also comparable is the percentage of disks with PAH emission: $3 \%$ in Serpens and $4 \%$ in Taurus.

In both regions, all disks with a $10 \mu \mathrm{m}$ silicate feature also show the $20 \mu \mathrm{m}$ one. However, a difference is found for disks with only the $20 \mu \mathrm{m}$ feature in emission: they amount to $17 \%$ of the disk population in Serpens and only $4.5 \%$ in Taurus. These statistics are summarized in Table 4.

IRS data on the 85 disk sources presented in Furlan et al. (2006) were obtained from the Spitzer archive and reduced in the

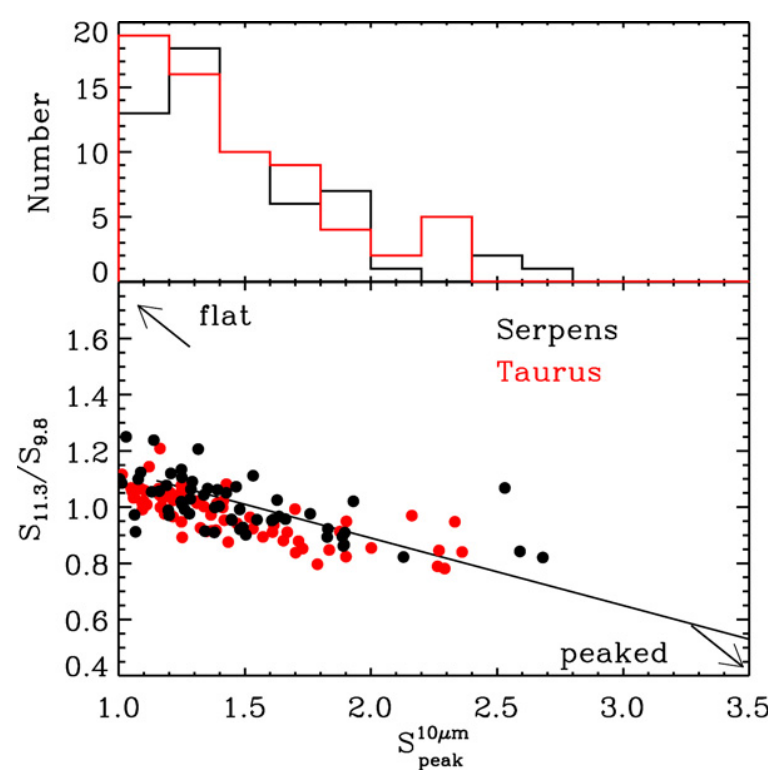

Figure 15. Top: distribution of the $10 \mu \mathrm{m}$ peak strength for Serpens (black) and Taurus (red). Bottom: strength and shape of the $10 \mu \mathrm{m}$ silicate feature for both Serpens (black) and Taurus (red).

(A color version of this figure is available in the online journal.)

Table 4

Statistics of the YSO Populations in Serpens and Taurus

\begin{tabular}{lccccc}
\hline \hline \multirow{2}{*}{ Cloud } & \multirow{2}{*}{ Embedded } & \multicolumn{4}{c}{ Disks } \\
\cline { 3 - 6 } & & PAH & Both 10 and 20 $\mu \mathrm{m}$ & Only 20 $\mu \mathrm{m}$ & Featureless \\
\hline Serpens & $18.3 \%$ & $2.6 \%$ & $60.0 \%$ & $13.9 \%$ & $4.3 \%$ \\
Taurus & $20.1 \%$ & $2.9 \%$ & $57.6 \%$ & $3.6 \%$ & $18.7 \%$ \\
\hline
\end{tabular}

same manner as our Serpens data. The same method described in Section 3.3.4 was applied to these data in order to compare the processes affecting the dust in both regions. Figure 15 (bottom) shows the strength versus shape of the $10 \mu \mathrm{m}$ silicate feature for both Serpens (black dots) and Taurus (red dots), as well as the distribution of peak strength for both regions (top). Serpens and Taurus present remarkably similar distributions, with populations clustered around flatter features and bigger grains, and almost no strongly peaked silicate emission sources. A KS test shows that the hypothesis that the distributions are drawn from the same population cannot be rejected (82\%).

\subsection{Implications}

Comparisons between disks around stars that were formed in clusters or in isolation in the Serpens Cloud, explored in Section 4.1, indicate very similar populations. Thus, statistically no differences are found in terms of both disk geometry (probed by the ratio $F_{30} / F_{13}$ ) and processes affecting the dust (probed by the silicate features). This suggests that local environment does not affect the evolution path and timescale of disks.

Even more remarkable is the agreement between the YSO populations of Serpens and Taurus, as well as the c2d IRS sample spread over five clouds, in terms of silicate features, as shown in Sections 4.2 and 4.3. Even though there are clear differences in silicate features from source to source within a cloud, the overall distribution of feature shapes is statistically indistinguishable: in all three samples, each containing of the order of 100 disks, the bulk of the sources has a rather flat silicate profile, and a tail of peaked shapes. 
If the difference in median ages is significant, the similarity seen in Figure 15 indicates that a 2-3 Myr difference in age is not reflected in a concurrent evolution of the average disk surface dust properties. This indicates that the dust population in the disk surface is an equilibrium between dust growth and destruction processes, which is maintained at least as long as the disk is optically thick.

The process of grain growth through coagulation and settling to the midplane has been shown to be much too short to be consistent with disk observations (Weidenschilling 1980; Dullemond \& Dominik 2005). That means that the small grain population must be replenished somehow, which could happen by fragmentation of bigger aggregates and turbulent mixing. It is widely accepted in the literature that the $10 \mu \mathrm{m}$ silicate feature is representative of the dust in the surface layers of the disk at a few AU from the star (Kessler-Silacci et al. 2007). Significant evolution could still take place in the disk mid-plane, which is not traced with these observations. Indeed, millimeter observations have indicated that disk midplanes are abundant in grains with $\mathrm{mm} / \mathrm{cm}$ sizes (Natta et al. 2004; Rodmann et al. 2006; Lommen et al. 2007). That means that, if this population of small grains in the disk surface must be replenished by fragmentation of bigger grains followed by turbulent mixing, the surface dust is an indirect tracer of midplane grains.

If the age difference between Serpens and Taurus is significant, then there exists a statistical equilibrium of processes of dust coagulation and fragmentation in the disk lasting at least a few million years that is independent of which YSO population is being studied. The observable IR dust characteristics of a cluster population of protoplanetary disks do not appear to depend on cluster properties (age, density) within the first $5 \mathrm{Myr}$, for relatively low-mass clusters. No specific property or event that may influence the infrared dust signatures of a single disk (stellar luminosity, spectral type, presence of a companion, disk-planet interactions, disk instabilities, etc.) produces detectable temporal or spatial evolutionary effects visible in the distribution of disk properties of an entire cluster within this time frame.

This is consistent with a picture in which a specific disk may change its appearance on short timescales (much less than $1 \mathrm{Myr}$ ), but in a reversible way. That is, the properties of disk surfaces may oscillate between different states (Bary et al. 2007; Muzerolle et al. 2009). If some evolution of the disk surface is irreversible, and happens at a given rate, a stable distribution of surface properties would not be seen over time. This requires that the effect on the disk surface of any reprocessing events has to be erased.

Another option is that the disk surface properties are determined by a single parameter, such as the initial conditions of the formation of such disks. In this scenario, those properties should be kept "frozen" over the observed timescale of $\sim$ few Myr. However, this cannot be the case for objects like EX Lup, where real time changes of the disk surface properties after the initial formation of the disk have been observed on timescales of just a few years (namely crystallization through thermal annealing of the dust in the disk surface, Ábrahám et al. 2009).

For this theory to work, the putative oscillation of states proposed here must be stable over disk lifetimes of $\sim 5 \mathrm{Myr}$. As long as the disks are gas-rich and optically thick, as in the three samples studied here, we do not see a distinguishable evolution. It will be important to search for surface evolution indicators in even older clusters. Such comparisons will yield constraints on the global timescale of disk evolution, which in turn will help constrain the importance of the processes that play a vital role in the dissipation of the disks.

The scenario in which a considerable population of small particles is still present after bigger aggregates are formed and altered through multiple events is consistent with evidence from primitive chondrites in our own solar system. Chondritic meteorites are observed to contain fine-grained dust-like matrices formed after coarse-grained materials such as chondrules and calcium-aluminum-rich inclusions (CAIs). This indicates that the solar nebula underwent violent reprocessing events in the feeding zones of parent bodies at 3-4 AU (see Pontoppidan \& Brearley (2010) for a recent review on solar system dust in an astrophysical context). These events included systemic melting of large dust aggregates and possibly even evaporation and re-condensation of silicate grains. In fact, presolar material is present in primitive chondrites only at the trace level: $\sim 100 \mathrm{ppm}$ (Lodders \& Amari 2005; Zinner et al. 2007), testifying to the complete evaporative destruction of ISM dust at a few AU in protoplanetary dust.

\section{CONCLUSIONS}

We present Spitzer/IRS spectra from a complete and unbiased flux limited sample of IR excess sources found in the Serpens Molecular Cloud, following the c2d mapping of this region.

1. Among our total of 147 IRS spectra, 22\% are found to be background contamination (including background stars, redshifted galaxies, and a planetary nebula candidate). This high number is not surprising given the position of Serpens, close to the galactic plane.

2. Excluding the background objects from the sample, the bona fide set of YSOs amounts to 115 objects. The embedded disk source ratio is $18 \%$, in agreement with the ratios derived from photometry (Evans et al. 2009) for the five c2d clouds.

3. Disks with PAH in emission amount to $2 \%$ of the YSO population, but $3 \%$ of the disk population. Only $\mathrm{G}$ and A stars show PAH emission. In the disk population, 73\% show both silicate emission features, at 10 and $20 \mu \mathrm{m}$, while $17 \%$ show only the $20 \mu \mathrm{m}$ feature. $4 \%$ of the disks show featureless mid-IR spectra. Only one source, 120, shows both PAH and silicate in emission.

4. Our YSO population in Serpens is very similar to that in Taurus, also studied with IRS spectra. In both regions about $70 \%$ of the disk sources present both 10 and $20 \mu \mathrm{m}$ silicate features in emission and the $10 \mu \mathrm{m}$ feature is never seen without the $20 \mu \mathrm{m}$ feature. The only significant difference between the two populations is in the number of sources lacking the $10 \mu \mathrm{m}$ feature but showing the $20 \mu \mathrm{m}$ feature. This may be indicative of small holes $(\lesssim 1 \mathrm{AU})$ in these sources.

5. The silicate features in the IRS spectra measure the grain sizes that dominate the mid-IR opacity. The relationships between shape and strength of these features present distributions very similar to those obtained from other large samples of young stars. Comparison with the models of Olofsson et al. (2009) yields grains consistent with sizes larger than a few $\mu \mathrm{m}$.

6. No significant differences are found in the disk geometry or the grain size distribution in the upper layers of circumstellar disks (probed by the silicate features) in clustered or 

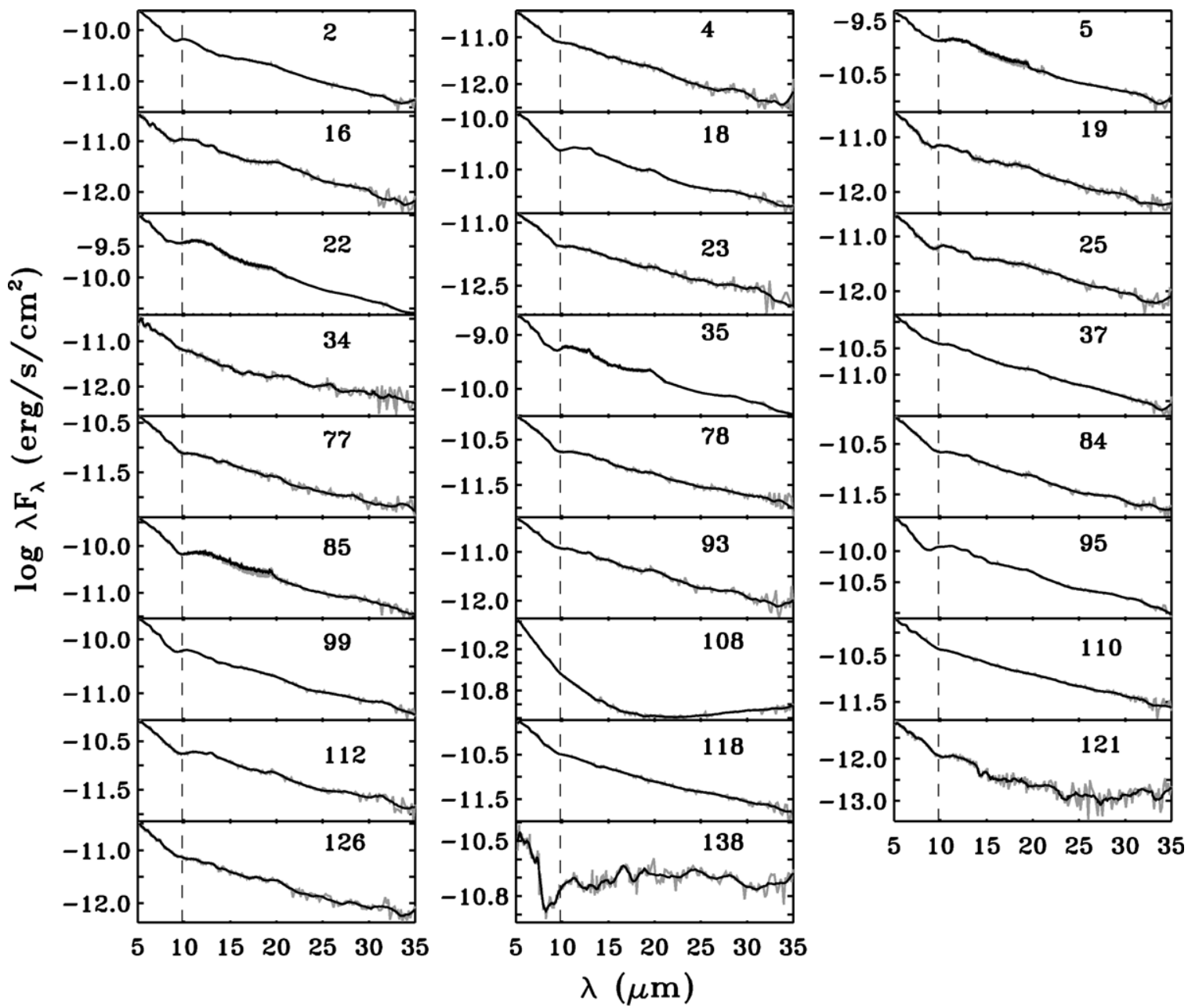

Figure 16. IRS spectra of the background stars toward Serpens with the characteristic $10 \mu \mathrm{m}$ silicate absorption feature and falling SED. In gray are the original spectra, while in black a binned version is overplotted. The object number is indicated at the top right of each panel.

field stars in the cloud, indicating that the local environment where a star is born does not influence the evolution of its harboring disk.

7. Quantitatively, the shape and strength of the $10 \mu \mathrm{m}$ silicate feature were used to compare both Serpens and Taurus as well as a large sample of disks across five clouds, indicating remarkably similar populations. This implies that the dust population in the disk surface results from an equilibrium between dust growth and destruction processes that are maintained over a few million years.

Support for this work, part of the Spitzer Space Telescope Legacy Science Program, was provided by NASA through Contract Numbers 1256316,1224608 , and 1230780 issued by the Jet Propulsion Laboratory, California Institute of Technology under NASA contract 1407 and by the Spanish Grant AYA 2005-0954. Astrochemistry at Leiden is supported by a Spinoza grant from the Netherlands Organization for Scientific Research (NWO) and by the Netherlands Research School for Astronomy (NOVA) grants. Support to K.M.P. was provided by NASA through Hubble Fellowship grant 01201.01, awarded by the Space Telescope Science Institute, which is operated by the Association of Universities for Research in Astronomy, Inc., for NASA, under contract NAS 5-26555. The authors are very grateful to L. Allamandola, R. Overzier, P. Beirão, R. Demarco, J. Green, and A. Kóspal for fruitful discussions, and would like to acknowledge the anonymous referee for suggestions that have improved the manuscript considerably.

Facilities: Spitzer(IRS)

\section{APPENDIX}

\section{BACKGROUND SOURCES}

Here the background contamination discussed in Section 3.1 is presented in three categories.

\section{A.1. Background Stars}

The IRS spectra of bright background stars, when seen through a molecular cloud, show the silicate feature at $10 \mu \mathrm{m}$ in absorption and not in emission as for disk sources (see Section 3.3.3), on top of a falling infrared spectrum. Oliveira et al. (2009) identified candidate background stars based on optical spectra for 78 objects in this sample. After determining spectral types, extinctions and luminosities, those objects were placed in a Hertzsprung-Russell diagram (HRD) and compared with two sets of isochrones and mass tracks (Baraffe et al. 1998, Siess et al. 2000). 20 out of those 78 objects proved to be much too luminous to be at the distance of Serpens $(d=259 \pm 13 \mathrm{pc}$, Straizys et al. 1996). Those objects were then classified as background sources, typically asymptotic giant branch (AGB) stars with dusty shells. Interestingly, 18 of the 20 objects classified as background in Oliveira et al. (2009) have 


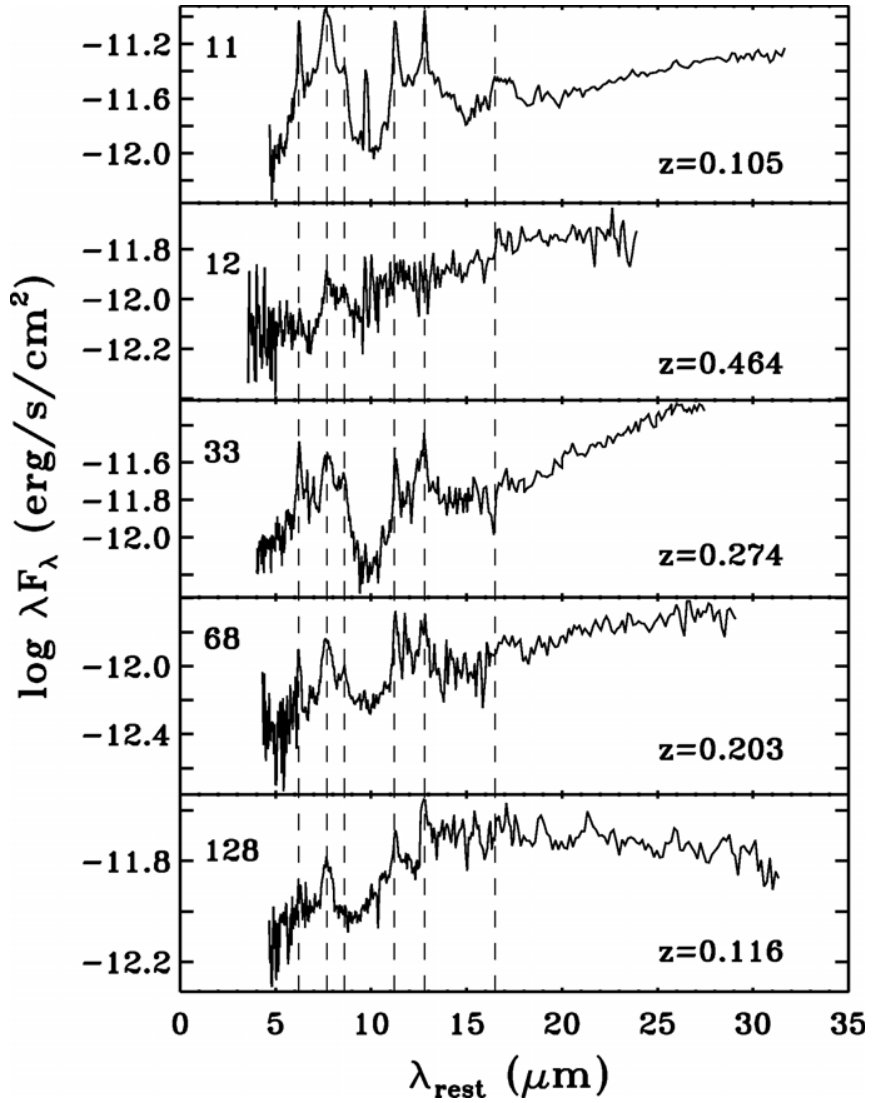

Figure 17. IRS spectra of the background galaxies toward Serpens, shown in rest-frame wavelength. The dashed lines indicate the rest-frame $\mathrm{PAH}$ features at $6.2,7.7,8.6,11.2,12.8$, and $16.4 \mu \mathrm{m}$. The object number and $z$ are indicated in each panel.

IRS spectra characteristic of background sources; the other two objects, 81 and 86, show silicates in emission (just like those seen in circumstellar disks) and were then re-classified as YSOs.

Besides these 18 objects, another 8 objects in this sample with falling mid-IR spectra show the $10 \mu \mathrm{m}$ silicate feature in absorption (objects 19, 25, 37, 78, 95, 126 were not observed by Oliveira et al. 2009), leading to their classification as background stars. Alternatively, those eight objects could be highly extincted YSOs, with silicate features in absorption arising from such extinction. However, using the dense cloud dust properties, as in Section 3.3.5, an extinction of $10 \mathrm{mag}$ would produce a $\tau_{9.7}$ of only 0.14 (see also Chiar et al. 2007). Since these objects do have silicate features in absorption, it is understood that they have high extinction (considerably higher than what is found for the cloud at their location). Thus, it is unlikely that they are actually highly extincted cloud members. Our working assumption is that these are background objects. These 26 spectra are shown in Figure 16.

\section{A.2. Background Galaxies}

Five of the sources with clear PAH features show wavelength shifts in their line positions compared with galactic sources. These features thus allow us to identify them as background galaxies in this sample. PAHs are common features in most nearby galaxies with ongoing or recent star formation (Smith et al. 2007), and some contamination by background galaxies is expected (Papovich et al. 2004).

By identifying PAH features from their shape and pattern (Tielens 2008 and references therein), the observed wavelength

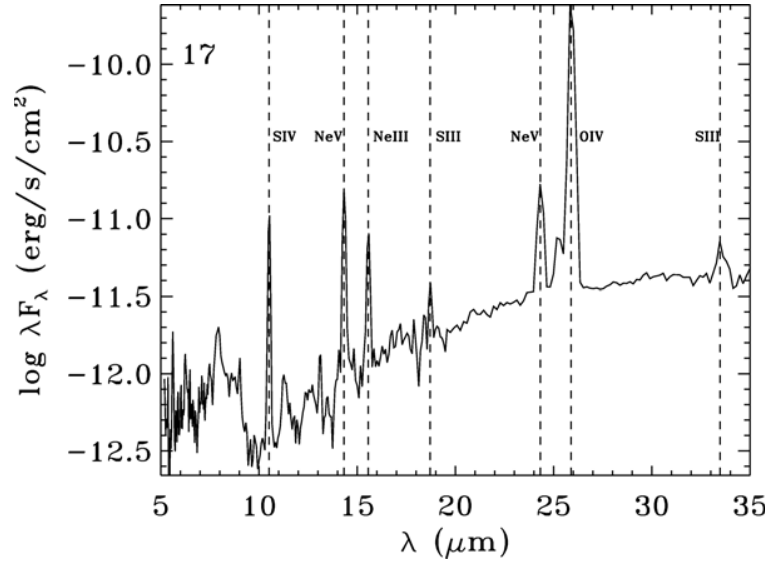

Figure 18. IRS spectra of the planetary nebula candidate (17) found among this sample, presenting high ionization lines.

Table 5

Characteristics of the Background Galaxies

\begin{tabular}{lccccc}
\hline \hline No. & $z$ & $24 \mu \mathrm{m}(\mathrm{Jy})$ & SFR $\left(M_{\odot} \mathrm{yr}^{-1}\right)$ & $L(\mathrm{TIR})\left(L_{\odot}\right)$ & Type \\
\hline 11 & 0.1051 & 0.01800 & 16 & $1.5 \times 10^{11}$ & LIRG \\
$12^{\mathrm{a}}$ & 0.4637 & 0.00893 & 731 & $4.8 \times 10^{12}$ & ULIRG \\
33 & 0.2742 & 0.01490 & 217 & $1.6 \times 10^{12}$ & ULIRG \\
68 & 0.2032 & 0.00909 & 47 & $4.0 \times 10^{11}$ & LIRG \\
128 & 0.1164 & 0.01010 & 11 & $1.1 \times 10^{11}$ & LIRG \\
\hline
\end{tabular}

Note. ${ }^{a}$ NVSS J182817+001604.

$\left(\lambda_{\text {obs }}\right)$ can be related to the rest-frame wavelength $\left(\lambda_{\text {rest }}\right)$ of each feature through a redshift $\left(z=\frac{\lambda_{\text {obs }}}{\lambda_{\text {rest }}}-1.0\right)$. Between 3 (12) and 5 (11 and 68) PAH features are identified in each spectrum, and the redshift of each line is calculated. For a given object, the $z$ values derived from the various lines agree within $2 \%$ and the mean of these values is taken as the redshift for that source. These five redshifted galaxies can be seen, in rest-frame wavelength, in Figure 17.

Star-forming galaxies show strong correlations between the extinction-corrected Paschen $\alpha$ luminosity coming from H II regions and the luminosity at $24 \mu \mathrm{m}$ due to the emission of dust heated by hot, young stars. Rieke et al. (2009) obtained powerlaw fits to the relation between the star formation rate (SFR) and the observed $24 \mu \mathrm{m}$ flux densities $\left(f_{24, \mathrm{obs}}\right)$, parameterized with respect to $z$ to take into account the fact that the restframe wavelengths sampled change with redshift (Equation (14) in their paper). In order to estimate the infrared luminosity $(L(T I R))$, we use their best-fit relations between the (rest-frame) $24 \mu \mathrm{m}$ luminosity, L(TIR), and SFR (Equations (11) and (25)).

We have calculated SFRs ${ }^{9}$ and $L$ (TIR) for the five background galaxies contaminating this sample. These results can be found in Table 5. $L(\mathrm{TIR})$ ranges from a few times $10^{11}$ to a few times $10^{12} L_{\odot}$, indicating that these galaxies are typical socalled luminous infrared galaxies (LIRGs, $L\left(\right.$ TIR) $>10^{11} L_{\odot}$ ), while two are ultra-luminous (ULIRGs, $L\left(\right.$ TIR) $>10^{12} L_{\odot}$ ). The latter are a class of heavily obscured galaxies having enormous instantaneous SFRs that are often associated with merging galaxies (Sanders \& Mirabel 1996). These galaxies are very rare locally and become more common at high redshift. Their space density rises by about a factor of 10 from $z=0$ to $z \sim 0.5$ (Magnelli et al. 2009), consistent with the relatively high redshifts found $(z \sim 0.3-0.5)$. Furthermore, due to its high

\footnotetext{
9 The SFRs correspond to an IMF according to Chabrier (2003).
} 
SFR and the empirical correlation between the infrared and radio emission from starburst galaxies (Condon 1992), the ULIRG 12 at $z=0.46$ can be identified with a previously unidentified radio source in the NRAO VLA Sky Survey (NVSS J182817+001604; Condon et al. 1998).

The inferred SFRs range between 11 and $731 M_{\odot} \mathrm{yr}^{-1}$, compared to a Milky Way SFR of about 3-5 $M_{\odot} \mathrm{yr}^{-1}$ (Prantzos $\&$ Aubert 1995, and references therein). For further reference, the SFR of Serpens is $5.7 \times 10^{-5} M_{\odot} \mathrm{yr}^{-1}$ (Evans et al. 2009). Even though the star formation observed in those galaxies is due to high-mass star-forming regions such as Orion, the high SFRs in the LIRGs and ULIRGs found are equivalent to $10^{5}-10^{7}$ molecular clouds such as Serpens!

\section{A.3. High Ionization Object}

One of the objects, 17 (SSTc2dJ18282720+0044450), in this sample presents very strong emission lines that do not seem compatible with YSOs. Figure 18 shows a blow-up of the spectrum, identifying the high ionization lines at $z=0$. The source itself is highly reddened, being barely detected in IRAC1 $(3.5 \mu \mathrm{m})$ but very prominent in MIPS1 $(24 \mu \mathrm{m})$, with a 3-24 $\mu \mathrm{m}$ slope of 1.82. Two types of galactic objects show such high excitation lines: dusty planetary nebulae and supernova remnants. The IRS spectrum of this object (17) resembles those of shocked ejecta of supernova remnants, as in Sandstrom et al. (2009) and Ghavamian et al. (2009) and that of planetary nebulae such as LMC 78, shown in Bernard-Salas et al. (2009).

The spectrum also shows PAH emission at 6.2, 7.7, 11.2, and $12.8 \mu \mathrm{m}$. Optical and near-IR spectra are necessary to confirm the nature of this object.

\section{REFERENCES}

Ábrahám, P., et al. 2009, Nature, 459, 224

Acke, B., \& van den Ancker, M. E. 2004, A\&A, 426, 151

Alexander, R. D. 2008, MNRAS, 391, L64

Alexander, R. D., Clarke, C. J., \& Pringle, J. E. 2006, MNRAS, 369, 216

Apai, D., Pascucci, I., Bouwman, J., Natta, A., Henning, T., \& Dullemond, C. P. 2005, Science, 310, 834

Baraffe, I., Chabrier, G., Allard, F., \& Hauschildt, P. H. 1998, A\&A, 337, 403

Baraffe, I., Chabrier, G., \& Gallardo, J. 2009, ApJ, 702, L27

Bary, J. S., Leisenring, J. M., \& Skrutskie, M. F. 2007, BAAS, 38, 1007

Bernard-Salas, J., Peeters, E., Sloan, G. C., Gutenkunst, S., Matsuura, M., Tielens, A. G. G. M., Zijlstra, A. A., \& Houck, J. R. 2009, ApJ, 699, 1541 Boogert, A. C. A., et al. 2004, ApJS, 154, 359

Boogert, A. C. A., et al. 2008, ApJ, 678, 985

Bottinelli, S., et al. 2010, ApJ, submitted

Bouwman, J., Lawson, W. A., Dominik, C., Feigelson, E. D., Henning, T., Tielens, A. G. G. M., \& Waters, L. B. F. M. 2006, ApJ, 653, L57

Bouwman, J., Meeus, G., de Koter, A., Hony, S., Dominik, C., \& Waters, L. B. F. M. 2001, A\&A, 375, 950

Bouwman, J., et al. 2008, ApJ, 683, 479

Brauer, F., Dullemond, C. P., \& Henning, T. 2008, A\&A, 480, 859

Brauer, F., Dullemond, C. P., Johansen, A., Henning, T., Klahr, H., \& Natta, A. 2007, A\&A, 469, 1169

Brown, J. M., et al. 2007, ApJ, 664, L107

Chabrier, G. 2003, PASP, 115, 763

Chapman, N. L., \& Mundy, L. G. 2009, ApJ, 699, 1866

Chapman, N. L., Mundy, L. G., Lai, S.-P., \& Evans, N. J. 2009, ApJ, 690, 496

Chiar, J. E., et al. 2007, ApJ, 666, L73

Clarke, C. J., Gendrin, A., \& Sotomayor, M. 2001, MNRAS, 328, 485

Condon, J. J. 1992, ARA\&A, 30, 575

Condon, J. J., Cotton, W. D., Greisen, E. W., Yin, Q. F., Perley, R. A., Taylor, G. B., \& Broderick, J. J. 1998, AJ, 115, 1693

Djupvik, A. A., André, P., Bontemps, S., Motte, F., Olofsson, G., Gålfalk, M., \& Florén, H.-G. 2006, A\&A, 458, 789

Dominik, C., \& Tielens, A. G. G. M. 1997, ApJ, 480, 647

Draine, B. T. 2003, ARA\&A, 41, 241

Dullemond, C. P., \& Dominik, C. 2004, A\&A, 421, 1075

Dullemond, C. P., \& Dominik, C. 2005, A\&A, 434, 971
Dullemond, C. P., \& Dominik, C. 2008, A\&A, 487, 205

Dullemond, C. P., Henning, T., Visser, R., Geers, V. C., van Dishoeck, E. F., \& Pontoppidan, K. M. 2007, A\&A, 473, 457

Eiroa, C., \& Casali, M. M. 1992, A\&A, 262, 468

Eiroa, C., Torrelles, J. M., Curiel, S., \& Djupvik, A. A. 2005, AJ, 130, 643

Evans, N. J., II, et al. 2003, PASP, 115, 965

Evans, N. J., II, et al. 2007, Delivery Documentation, http://ssc.spitzer.caltech. edu/spitzermission/observingprograms/legacy/c2d/

Evans, N. J., et al. 2009, ApJS, 181, 321

Fazio, G. G., et al. 2004, ApJS, 154, 10

Furlan, E., et al. 2006, ApJS, 165, 568

Furlan, E., et al. 2008, ApJS, 176, 184

Geers, V. C. 2007, Ph.D. thesis, Leiden University

Geers, V. C., et al. 2006, A\&A, 459, 545

Ghavamian, P., Raymond, J. C., Blair, W. P., Long, K. S., Tappe, A., Park, S., \& Winkler, P. F. 2009, ApJ, 696, 1307

Gorti, U., \& Hollenbach, D. 2009, ApJ, 690, 1539

Greene, T. P., Wilking, B. A., Andre, P., Young, E. T., \& Lada, C. J. 1994, ApJ, 434, 614

Haisch, K. E., Jr., Lada, E. A., \& Lada, C. J. 2001, ApJ, 553, L153

Hartmann, L., Ballesteros-Paredes, J., \& Bergin, E. A. 2001, ApJ, 562, 852

Harvey, P., Merín, B., Huard, T. L., Rebull, L. M., Chapman, N., Evans, N. J., II., \& Myers, P. C. 2007, ApJ, 663, 1149

Harvey, P. M., et al. 2006, ApJ, 644, 307

Harvey, P. M., et al. 2007, ApJ, 663, 1139

Hillenbrand, L. A. 2008, Phys. Scr. T, 130, 014024

Hillenbrand, L. A. 2009, in IAU Symp. 258, The Ages of Stars, ed. E. E. Mamajek, D. R. Soderblom, \& R. F. G. Wyse (Cambridge: Cambridge Univ. Press), 81

Houck, J. R., et al. 2004, ApJS, 154, 18

Ireland, M. J., \& Kraus, A. L. 2008, ApJ, 678, L59

Johansen, A., Brauer, F., Dullemond, C., Klahr, H., \& Henning, T. 2008, A\&A, 486,597

Jørgensen, J. K., Johnstone, D., Kirk, H., Myers, P. C., Allen, L. E., \& Shirley, Y. L. 2008, ApJ, 683, 822

Kaas, A. A., et al. 2004, A\&A, 421, 623

Kenyon, S. J., \& Hartmann, L. 1987, ApJ, 323, 714

Kenyon, S. J., \& Hartmann, L. 1995, ApJS, 101, 117

Kessler-Silacci, J., et al. 2006, ApJ, 639, 275

Kessler-Silacci, J. E., et al. 2007, ApJ, 659, 680

Lada, C. J. 1987, Star Forming Regions, 115, 1

Lada, E. A., \& Lada, C. J. 1995, AJ, 109, 1682

Lahuis, F., et al. 2006, c2d Spectroscopy Explanatory Supplement, Technical Report (Pasadena, CA: Spitzer Science Center)

Lodders, K., \& Amari, S. 2005, Geochemistry, 65, 93

Lommen, D., et al. 2007, A\&A, 462, 211

Magnelli, B., Elbaz, D., Chary, R. R., Dickinson, M., Le Borgne, D., Frayer, D T., \& Willmer, C. N. A. 2009, A\&A, 496, 57

McClure, M. 2009, ApJ, 693, L81

Meeus, G., Bouwman, J., Dominik, C., Waters, L. B. F. M., \& de Koter, A. 2002, A\&A, 392, 1039

Meeus, G., Waters, L. B. F. M., Bouwman, J., van den Ancker, M. E., Waelkens, C., \& Malfait, K. 2001, A\&A, 365, 476

Merín, B., et al. 2007, ApJ, 661, 361

Merín, B., et al. 2010, ApJ, submitted

Muzerolle, J., Hillenbrand, L., Calvet, N., Briceño, C., \& Hartmann, L. 2003, ApJ, 592, 266

Muzerolle, J., et al. 2009, ApJ, 704, L15

Natta, A., Testi, L., Muzerolle, J., Randich, S., Comerón, F., \& Persi, P. 2004, A\&A, 424, 603

Natta, A., Testi, L., Neri, R., Shepherd, D. S., \& Wilner, D. J. 2004, A\&A, 416, 179

Naylor, T. 2009, MNRAS, 399, 432

Öberg, K. I., Boogert, A. C. A., Pontoppidan, K. M., Blake, G. A., Evans, N. J., Lahuis, F., \& van Dishoeck, E. F. 2008, ApJ, 678, 1032

Oliveira, I., et al. 2009, ApJ, 691, 672

Olofsson, J., et al. 2009, A\&A, 507, 327

Papovich, C., et al. 2004, ApJS, 154, 70

Pascucci, I., Apai, D., Luhman, K., Henning, T., Bouwman, J., Meyer, M. R., Lahuis, F., \& Natta, A. 2009, ApJ, 696, 143

Pontoppidan, K. M., \& Brearly, A. J. 2010, in Protoplanetary Dust, ed. D. Apai $\&$ D. S. Lauretta (Cambridge: Cambridge Univ. Press)

Pontoppidan, K. M., Dullemond, C. P., van Dishoeck, E. F., Blake, G. A., Boogert, A. C. A., Evans, N. J., II, Kessler-Silacci, J. E., \& Lahuis, F. 2005, ApJ, 622, 463

Pontoppidan, K. M., et al. 2008, ApJ, 678, 1005

Prantzos, N., \& Aubert, O. 1995, A\&A, 302, 69 
Przygodda, F., van Boekel, R., Àbrahàm, P., Melnikov, S. Y., Waters, L. B. F. M., \& Leinert, C. 2003, A\&A, 412, L43

Quillen, A. C., Blackman, E. G., Frank, A., \& Varnière, P. 2004, ApJ, 612, L137

Richling, S., \& Yorke, H. W. 1998, A\&A, 340, 508

Richling, S., \& Yorke, H. W. 2000, ApJ, 539, 258

Rieke, G. H., Alonso-Herrero, A., Weiner, B. J., Pérez-González, P. G., Blaylock, M., Donley, J. L., \& Marcillac, D. 2009, ApJ, 692, 556

Rieke, G. H., et al. 2004, ApJS, 154, 25

Rodmann, J., Henning, T., Chandler, C. J., Mundy, L. G., \& Wilner, D. J. 2006, A\&A, 446, 211

Sanders, D. B., \& Mirabel, I. F. 1996, ARA\&A, 34, 749

Sandstrom, K., Bolatto, A., Stanimirović, S., van Loon, J. Th., \& Smith, J. D. T. 2009, ApJ, 696, 2138

Sargent, B. A., et al. 2009, ApJS, 182, 477

Savitzky, A., \& Golay, M. J. E. 1964, Anal. Chem., 36, 1627

Siess, L., Dufour, E., \& Forestini, M. 2000, A\&A, 358, 593

Smith, J. D. T., et al. 2007, ApJ, 656, 770

Sterzik, M. F., Morfill, G. E., \& Dubrulle, B. 1995, Ap\&SS, 224, 567

Straizys, V., Cernis, K., \& Bartasiute, S. 1996, Baltic Astron., 5, 125

Strom, S. W. 1992, Nature, 359, 360
Testi, L., \& Sargent, A. I. 1998, ApJ, 508, L91

Tielens, A. G. G. M. 2008, ARA\&A, 46, 289

Uchida, K. I., et al. 2004, ApJS, 154, 439

van Boekel, R., Waters, L. B. F. M., Dominik, C., Bouwman, J., de Koter, A., Dullemond, C. P., \& Paresce, F. 2003, A\&A, 400, L21

van Boekel, R., Waters, L. B. F. M., Dominik, C., Dullemond, C. P., Tielens, A. G. G. M., \& de Koter, A. 2004, A\&A, 418, 177

Vandenbussche, B., Dominik, C., Min, M., van Boekel, R., Waters, L. B. F. M., Meeus, G., \& de Koter, A. 2004, A\&A, 427, 519

Varnière, P., Blackman, E. G., Frank, A., \& Quillen, A. C. 2006, ApJ, 640, 1110

Visser, R., Geers, V. C., Dullemond, C. P., Augereau, J.-C., Pontoppidan, K. M., \& van Dishoeck, E. F. 2007, A\&A, 466, 229

Watson, D. M., et al. 2009, ApJS, 180, 84

Weidenschilling, S. J. 1980, Icarus, 44, 172

Weingartner, J. C., \& Draine, B. T. 2001, ApJ, 548, 296

White, R. J., \& Basri, G. 2003, ApJ, 582, 1109

Winston, E., et al. 2007, ApJ, 669, 493

Winston, E., et al. 2009, AJ, 137, 4777

Zhang, C. Y., Laureijs, R. J., \& Clark, F. O. 1988, A\&A, 196, 236

Zinner, E., et al. 2007, Geochim. Cosmochim. Acta, 71, 4786 